\title{
Mixed-integer sets from two rows of two adjacent simplex bases
}

\author{
Kent Andersen $^{1} \quad$ Quentin Louveaux $^{2} \quad$ Robert Weismantel $^{1}$
}

December 9, 2009

\begin{abstract}
In [1] we studied a mixed-integer set arising from two rows of a simplex tableau. We showed that facets of such a set can be obtained from lattice point free triangles and quadrilaterals associated with either three or four variables. In this paper we generalize our findings and show that, when upper bounds on the non-basic variables are also considered, further classes of facets arise that cannot be obtained from triangles and quadrilaterals. Specifically, when exactly one upper bound on a non-basic variable is introduced, stronger inequalities that can be derived from pentagons involving up to six variables also appear.
\end{abstract}

Keywords Mixed Integer Programming, Valid Inequalities, Two Rows, Lattice-Point-Free Polyhedra

\section{Introduction}

The mixed-integer set considered in this paper is given by

$$
P_{\mathrm{I}}:=\left\{(x, s) \in \mathbb{Z}^{2} \times \mathbb{R}_{+}^{n}: x=f+\sum_{j \in N} s_{j} r^{j}, \text { and } s_{j} \leq u_{j} \text { for } j \in N\right\},
$$

where $N:=\{1,2, \ldots, n\}, f \in \mathbb{Q}^{2}, r^{j} \in \mathbb{Q}^{2}$ for $j \in N$ and $u_{j} \in \mathbb{R}_{+} \cup\{+\infty\}$ for $j \in N$. We partition $N$ into $N=B \cup U$ where $U$ is the index set for variables $s_{j}$ with $u_{j}=+\infty$. The set $P_{\mathrm{LP}}$ denotes the LP relaxation of $P_{I}$ and the $j^{\text {th }}$ unit vector in $\mathbb{R}^{n}$ is denoted $e_{j}$. We call the vectors $\left\{r^{j}\right\}_{j \in N}$ for rays, and we assume $r^{j} \neq 0$ for all $j \in N$.

Various attempts have been made to understand the polyhedral structure of $\operatorname{conv}\left(P_{\mathrm{I}}\right)$. Gomory's mixed integer cuts [6], mixed integer rounding cuts [10], lift-and-project cuts and split cuts $[3,4]$ are all valid for $\operatorname{conv}\left(P_{\mathrm{I}}\right)$. However, these classes of inequalities do not suffice to describe $\operatorname{conv}\left(P_{\mathrm{I}}\right)$. The reason is

${ }^{01}$ Otto-von-Guericke Universität Magdeburg, Institut für Mathematische Optimierung ${ }^{2}$ Université de Liège, Montefiore Institute 
that all these inequalities can be derived from a one-row relaxation of $\operatorname{conv}\left(P_{\mathrm{I}}\right)$, and this is not sufficient in order to characterize all valid inequalities for $\operatorname{conv}\left(P_{\mathrm{I}}\right)$ [4].

The polyhedron $\operatorname{conv}\left(P_{\mathrm{I}}\right)$ was introduced in [1] in the special case when $u_{j}=\infty$ for all $j \in N$, and all facets of $\operatorname{conv}\left(P_{\mathrm{I}}\right)$ were characterized geometrically: It was shown that all facets of $\operatorname{conv}\left(P_{\mathrm{I}}\right)$ could be derived from either one-row relaxations or lattice point free triangles and quadrilaterals associated with three or four non-basic variables respectively. Cornuéjols and Margot later characterized exactly which lattice point free triangles and quadrilaterals give rise to facets of $\operatorname{conv}\left(P_{\mathrm{I}}\right)$ [5]. Cornuéjols and Margot also related $\operatorname{conv}\left(P_{\mathrm{I}}\right)$ to the corner polyhedron introduced by Gomory $[7,8]$ by associating a variable $s_{r}$ with every vector $r \in \mathbb{Z}^{2}$, and then imposing finite support on the set of variables that are positive in a feasible solution. The key result in both papers $[1,5]$ is to provide a bijective map between the facets of $\operatorname{conv}\left(P_{\mathrm{I}}\right)$ and certain two-dimensional lattice point free triangles and quadrilaterals.

In this paper we explore the geometric structure of $\operatorname{conv}\left(P_{\mathrm{I}}\right)$ when upper bounds are present, i.e., when we have $B \neq \emptyset$. It turns out that upper bounds substantially complicate the structure of $\operatorname{conv}\left(P_{\mathrm{I}}\right)$. In the special case of exactly one upper bound on a non-basic variable, we provide a complete description of the lattice point free polygons associated with the facet defining inequalities for $\operatorname{conv}\left(P_{\mathrm{I}}\right)$. Specifically, in this case, we show that a complete description of $\operatorname{conv}\left(P_{\mathrm{I}}\right)$ is available if pentagons obtained from up to six variables are considered in addition to triangles and quadrilaterals. Furthermore, in the obtained inequalities, the coefficient of the bounded variable is strictly stronger. If we wanted to obtain such an inequality using a standard triangle or quadrilateral, it would contain at least an integer point in its interior. This case is however interesting, since relaxations of this type can be obtained from two adjacent bases of the LP relaxation of a mixed integer program. Such relaxations might therefore be interesting computationally. Specifically, consider two rows of a simplex tableau with basic variables $\left(x_{1}, x_{2}\right) \in \mathbb{Z}^{2}$ and nonbasic variables $s \in \mathbb{R}_{+}^{n}$, and assume $\left(x_{1}^{\mathrm{B}}, x_{2}^{\mathrm{B}}\right) \notin \mathbb{Z}^{2}$, where $x^{\mathrm{B}}$ denotes the value of $x$ in the basic solution associated with the simplex tableau. Choose any edge in the polyhedron associated with the linear programming relaxation that connects the vertex $x^{\mathrm{B}}$ with a second vertex $x^{\mathrm{B}}$, where $B$ and $B^{\prime}$ are adjacent bases. We can now write $\left(x_{1}^{\mathrm{B}^{\prime}}, x_{2}^{\mathrm{B}^{\prime}}\right)=\left(x_{1}^{\mathrm{B}}, x_{2}^{\mathrm{B}}\right)+u_{i} r^{i}$, where $\left(x_{1}, x_{2}\right)=\left(x_{1}^{\mathrm{B}}, x_{2}^{\mathrm{B}}\right)+\sum_{j \in N} s_{j} r^{j}$ denotes the two rows of the simplex tableau associated with $B$ and $i \in N$. A natural mixed integer programming relaxation associated with the pivot along the edge from $x^{\mathrm{B}}$ to $x^{\mathrm{B}}$ is now the set $\left\{\left(x_{1}, x_{2}\right) \in \mathbb{Z}^{2}:\left(x_{1}, x_{2}\right)=\left(x_{1}^{\mathrm{B}}, x_{2}^{\mathrm{B}}\right)+\sum_{j \in N} s_{j} r^{j}, s \geq 0\right.$ and $\left.s_{i} \leq u_{i}\right\}$.

In the general case when several upper bounds are present, we have not been able to characterize all lattice point free polygons that arise from facet defining inequalities for $\operatorname{conv}\left(P_{\mathrm{I}}\right)$. An explicit and geometric construction of these polygons is an interesting open problem for future research.

The remainder of the paper is organized as follows. In Sect. 2 we give some basic polyhedral properties of $\operatorname{conv}\left(P_{\mathrm{I}}\right)$. In particular, we derive a general form of a facet defining inequalities for $\operatorname{conv}\left(P_{\mathrm{I}}\right)$. Representations of integer points 
$x \in \mathbb{Z}^{2}$ in terms of the non-basic variables are considered in Sect. 3. Finally we characterize the structure of the facets of $\operatorname{conv}\left(P_{\mathrm{I}}\right)$ in Sect. 4, where we show that pentagons suffice to derive facets for $\operatorname{conv}\left(P_{\mathrm{I}}\right)$ when only one upper bound is present.

\section{Basic polyhedral properties of $\operatorname{conv}\left(P_{\mathbf{I}}\right)$}

We now describe a number of structural properties of $\operatorname{conv}\left(P_{\mathrm{I}}\right)$. Many of these properties are generalizations of results in [1].

Observation 1 The set $\operatorname{conv}\left(P_{I}\right)$ has the following properties.

(i) If $\operatorname{dim}\left(\operatorname{cone}\left(\left\{r^{j}\right\}_{j \in U}\right)\right)=2$, then the dimension of $\operatorname{conv}\left(P_{I}\right)$ is $n$.

(ii) The extreme rays of $\operatorname{conv}\left(P_{I}\right)$ are $\left(r^{j}, e_{j}\right)$ for $j \in U$.

(iii) The vertices $(x, s)$ of $\operatorname{conv}\left(P_{I}\right)$ are such that the number of indices for which $0<s_{j}<u_{j}$ is at most two.

In the following, we study properties of the valid inequalities for $\operatorname{conv}\left(P_{\mathrm{I}}\right)$. We are interested in non-trivial valid inequalities, i.e., valid inequalities that are tight for at least a point $(\bar{x}, \bar{s}) \in P_{\mathrm{I}}$, and inequalities that are not conic combinations of the upper and lower bounds.

Lemma 1 Every non-trivial valid inequality for $P_{I}$ can be written as

$$
\sum_{i \in B_{-}} \alpha_{i}\left(u_{i}-s_{i}\right)+\sum_{i \in B_{+}} \alpha_{i} s_{i}+\sum_{i \in U} \alpha_{i} s_{i} \geq 1
$$

where $\alpha_{i} \geq 0$ for all $i$ and $\left(B_{-}, B_{+}\right)$is a partitioning of $B$.

Proof: Let $\sum_{j \in N} \alpha^{\prime \prime} s_{j} \geq \beta^{\prime \prime}$ be a non-trivial valid inequality for $\operatorname{conv}\left(P_{\mathrm{I}}\right)$ and let $(\bar{x}, \bar{s}) \in P_{\mathrm{I}}$ be a tight feasible point. From the fact that the vectors $\left(r^{j}, e_{j}\right)$ for $j \in U$ are the extreme rays of $\operatorname{conv}\left(P_{\mathrm{I}}\right)$, we conclude that $\alpha_{j}^{\prime \prime} \geq 0$ for all $j \in U$ (these non-negativity constraints are explicitly part of the inequality set that defines the polar of $\left.\operatorname{conv}\left(P_{\mathrm{I}}\right)\right)$.

Define $B_{-}:=\left\{j \in B \mid \alpha_{j}^{\prime \prime}<0\right\}$ and $B_{+}:=B \backslash B_{-}$. Also define $\alpha_{j}^{\prime}:=-\alpha_{j}^{\prime \prime}$ for $j \in B_{-}, \alpha_{j}^{\prime}:=\alpha_{j}^{\prime \prime}$ for $j \in N \backslash B_{-}$and $\beta^{\prime}:=\beta^{\prime \prime}-\sum_{j \in B_{-}} u_{j} \alpha_{j}^{\prime \prime}$. Then $\sum_{j \in B_{-}} \alpha_{j}^{\prime}\left(u_{j}-s_{j}\right)+\sum_{j \in N \backslash B_{-}} \alpha_{j}^{\prime} s_{j} \geq \beta^{\prime}$ is equivalent to $\sum_{j \in N} \alpha_{j}^{\prime \prime} s_{j} \geq \beta^{\prime \prime}$, and $\alpha_{j}^{\prime} \geq 0$ for all $j \in N$. Inserting the tight point $(\bar{x}, \bar{s})$ into this inequality, and observing that $\sum_{j \in B_{-}} \alpha_{j}^{\prime}\left(u_{j}-\bar{s}_{j}\right)+\sum_{j \in N \backslash B_{-}} \alpha_{j}^{\prime} \bar{s}_{j} \geq 0$, we can therefore not have $\beta^{\prime}<0$. Furthermore, if $\beta^{\prime}=0$, then the inequality is a trivial conic combination of the non-negativity constraints and the upper bounds which contradicts the assumption that the inequality is non-trivial. Hence, if we let $\alpha_{j}:=\frac{\alpha_{j}^{\prime}}{\beta^{\prime}}$, we obtain the desired form. 
We now interpret Lemma 1 in terms of the following sets that are isomorphic to $P_{I}$. Given $B_{-} \subseteq B$, let $B_{+}:=B \backslash B_{-}$and let $f\left(B_{-}\right):=f+\sum_{j \in B_{-}} u_{j} r^{j}$. The set

$$
\begin{aligned}
P_{\mathrm{I}}\left(B_{-}\right):= & \left\{(x, s) \in \mathbb{Z}^{2} \times \mathbb{R}_{+}^{n}:\right. \\
& x=f\left(B_{-}\right)+\sum_{i \in U} r^{i} s_{i}-\sum_{j \in B_{-}} r^{j} s_{j}+\sum_{j \in B_{+}} r^{j} s_{j} \text { and } \\
& \left.s_{j} \leq u_{j} \text { for } j \in B\right\}
\end{aligned}
$$

is isomorphic to $P_{I}$. Indeed, given any $(x, s) \in P_{I}$, the point $\left(x, s^{\prime}\right)$ with $s_{j}^{\prime}=s_{j}$ for $j \in B_{+} \cup U$ and $s_{j}^{\prime}=u_{j}-s_{j}$ for $j \in B_{-}$is in $P_{I}\left(B_{-}\right)$, and this mapping is oneto-one. Furthermore, from Lemma 1 it follows that an inequality $\sum_{j \in B_{-}} \alpha_{i}\left(u_{i}-\right.$ $\left.s_{i}\right)+\sum_{j \in B_{+}} \alpha_{j} s_{j}+\sum_{i \in U} \alpha_{i} s_{i} \geq 1$ with $\alpha_{i} \geq 0$ for $i \in B \cup U$ is valid for $P_{I}$ if and only if the inequality

$$
\sum_{i \in U} \alpha_{i} s_{i}+\sum_{j \in B} \alpha_{j} s_{j} \geq 1
$$

is valid for $P_{I}\left(B_{-}\right)$. Since the purpose in the remainder of this paper is to study the structure of an arbitrary non-trivial facet defining inequality for $P_{I}$, we may assume without loss of generality that this inequality is of the form (2). We call valid inequalities for $P_{I}$ of the form (2) in standard form, and we are interested in characterizing all non-trivial facet defining inequalities for $\operatorname{conv}\left(P_{\mathrm{I}}\right)$ that are in standard form. Observe, however, that to obtain all of the valid inequalities for $\operatorname{conv}\left(P_{\mathrm{I}}\right)$, every set $B_{-} \subseteq B$ must be considered. In other words, every basic feasible solution of $P_{\mathrm{LP}}$ needs to be examined.

We now associate a two-dimensional lattice point free polyhedron with a valid inequality $\sum_{j \in N} \alpha_{j} s_{j} \geq 1$ for $\operatorname{conv}\left(P_{\mathrm{I}}\right)$ in standard form. This polyhedron gives a two-dimensional geometric representation of the facets of $\operatorname{conv}\left(P_{I}\right)$.

Lemma 2 Let $\sum_{j \in N} \alpha_{j} s_{j} \geq 1$ be a valid inequality for $\operatorname{conv}\left(P_{I}\right)$ that is in standard form. Consider the following convex polyhedron in $\mathbb{R}^{2}$

$$
L_{\alpha}=\left\{x \in \mathbb{R}^{2}: \text { there exists } s \in \mathbb{R}^{n} \text { s.t. }(x, s) \in P_{L P} \text { and } \sum_{j \in N} \alpha_{j} s_{j} \leq 1\right\} .
$$

The interior of $L_{\alpha}$ does not contain any integer point.

Proof: If $\bar{x} \in \operatorname{interior}\left(L_{\alpha}\right)$, then there exists $\bar{s} \in \mathbb{R}_{+}^{n}$ such that $(\bar{x}, \bar{s}) \in P_{L P}$ and $\sum_{j \in N} \alpha_{j} \bar{s}_{j}<1$. Since $\sum_{j \in N} \alpha_{j} s_{j} \geq 1$ is valid for $P_{I}$, we can not have that $\bar{x}$ is integer.

Example 1: Consider the set

$$
\begin{aligned}
P_{\mathrm{I}}=\left\{(x, s) \in \mathbb{Z}^{2} \times \mathbb{R}_{+}^{4}: 0 \leq s_{e} \leq 1\right. \text { and } \\
\left.\quad x=\left(\begin{array}{c}
0.2 \\
0.2
\end{array}\right)+\left(\begin{array}{c}
0.7 \\
0
\end{array}\right) s_{e}+\left(\begin{array}{c}
-1 \\
1
\end{array}\right) s_{1}+\left(\begin{array}{c}
1 \\
1
\end{array}\right) s_{2}+\left(\begin{array}{c}
0 \\
-1
\end{array}\right) s_{3}\right\},
\end{aligned}
$$


and the inequality

$$
\frac{7}{30} s_{e}+s_{1}+\frac{5}{3} s_{2}+2 s_{3} \geq 1
$$

The corresponding set $L_{\alpha}$ is shown in Fig. 1. As seen from the figure, $L_{\alpha}$ does not contain integer points in its interior. Hence (4) is valid for $\operatorname{conv}\left(P_{\mathrm{I}}\right)$.

Note that, conversely, the coefficients $\alpha_{j}$ for $j=1, \ldots, 3$ can be obtained from the polygon $L_{\alpha}$ as follows: $\alpha_{j}$ is the ratio between the length of $r^{j}$ and the distance between $f$ and the intersection of $\left\{f+\lambda r^{j}: \lambda \geq 0\right\}$ with $L_{\alpha}$. The coefficient $\alpha_{e}$ is obtained by the ratio of the length of $r^{e}$ and the distance between $f$ and the intersection of the dotted lines on Fig. 1. We will see in the last section how to find the coefficients from the geometry in general.

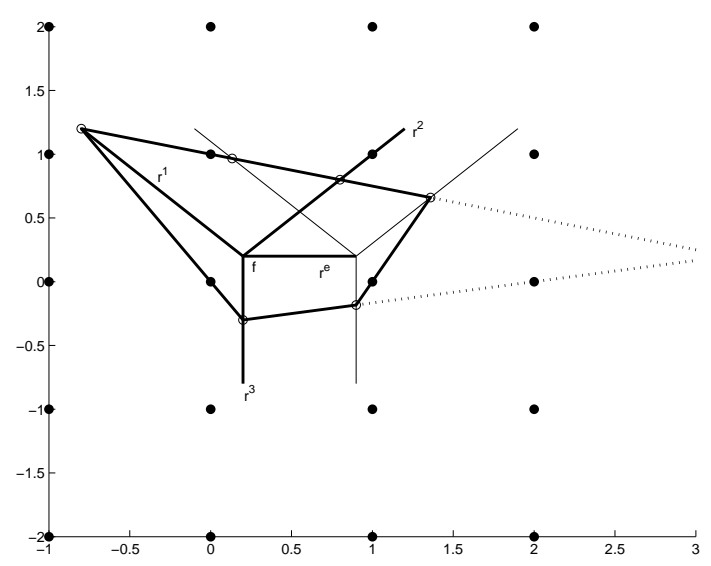

Figure 1: The set $L_{\alpha}$ for a valid inequality for $\operatorname{conv}\left(P_{\mathrm{I}}\right)$

The interior of $L_{\alpha}$ gives a two-dimensional representation of the points $x \in$ $\mathbb{R}^{2}$ affected by adding the inequality $\sum_{j \in N} \alpha_{j} s_{j} \geq 1$ to the LP relaxation $P_{\mathrm{LP}}$ of $P_{\mathrm{I}}$. In other words, for any $(x, s) \in P_{\mathrm{LP}}$ satisfying $\sum_{j \in N} \alpha_{j} s_{j}<1$, we have $x \in \operatorname{interior}\left(L_{\alpha}\right)$. Furthermore, for a facet defining inequality $\sum_{j \in N} \alpha_{j} s_{j} \geq 1$ for $\operatorname{conv}\left(P_{\mathrm{I}}\right)$, there exist $n$ affinely independent points $\left(x^{i}, s^{i}\right) \in P_{\mathrm{I}}, i=1,2, \ldots, n$, such that $\sum_{j \in N} \alpha_{j} s_{j}^{i}=1$. The integer points $\left\{x^{i}\right\}_{i \in N}$ are on the boundary of $L_{\alpha}$, i.e., they belong to the integer set

$$
X_{\alpha}:=\left\{x \in \mathbb{Z}^{2}: \exists s \in \mathbb{R}_{+}^{n} \text { s.t. }(x, s) \in P_{\mathrm{LP}} \text { and } \sum_{j \in N} \alpha_{j} s_{j}=1\right\} .
$$

We have $X_{\alpha}=L_{\alpha} \cap \mathbb{Z}^{2}$, and $X_{\alpha} \neq \emptyset$ whenever $\sum_{j \in N} \alpha_{j} s_{j} \geq 1$ defines a facet of $\operatorname{conv}\left(P_{\mathrm{I}}\right)$.

In the remainder of the paper we only consider inequalities for which $\alpha_{j}>0$ for all $j \in U$. The reason is the following result. 
Lemma 3 Any facet defining inequality $\sum_{j \in N} \alpha_{j} s_{j} \geq 1$ for $\operatorname{conv}\left(P_{I}\right)$ of the form (2) with a zero coefficient on some unbounded variable is a split cut. In other words, if $\alpha_{j}=0$ for some $j \in U$, then there exists $\left(\pi, \pi_{0}\right) \in \mathbb{Z}^{2} \times \mathbb{Z}$ such that $L_{\alpha} \subseteq\left\{\left(x_{1}, x_{2}\right): \pi_{0} \leq \pi_{1} x_{1}+\pi_{2} x_{2} \leq \pi_{0}+1\right\}$.

Lemma 3 was proven in [1] in the case when $B=\emptyset$. This proof also applies when bounded variables are present, so we do not repeat it here. We will only sketch the main ideas. The key observation is that, if $\alpha_{k}=0$ for some $k \in U$, then the line $\left\{f+\mu r^{k}: \mu \in \mathbb{R}\right\}$ is lattice point free and strictly contained in $L_{\alpha}$. Hence $r^{k}$ and $-r^{k}$ are extreme rays of $L_{\alpha}$, and therefore $L_{\alpha}$ is of the form $\left\{x \in \mathbb{R}^{2}: \pi_{0}^{1} \leq\left(\pi^{\prime}\right)^{T} x \leq \pi_{0}^{2}\right\}$ for some $\pi^{\prime} \in \mathbb{Z}^{2}$ and $\pi_{0}^{1}<\pi_{0}^{2}$. Since $L_{\alpha}$ is lattice point free, this implies there exists $\left(\pi, \pi_{0}\right) \in \mathbb{Z}^{3}$ such that $L_{\alpha}$ is contained in $\left\{x \in \mathbb{R}^{2}: \pi_{0} \leq \pi^{T} x \leq \pi_{0}+1\right\}$, which shows that $\sum_{j \in N} \alpha_{j} s_{j} \geq 1$ must be the split cut derived from $\left(\pi, \pi_{0}\right)$.

In the following we therefore assume $\alpha_{j}>0$ for all $j \in U$. Clearly $\operatorname{conv}\left(X_{\alpha}\right)$ is a convex polygon with only integer vertices, and since $X_{\alpha} \subseteq L_{\alpha}, \operatorname{conv}\left(X_{\alpha}\right)$ does not have any integer point in its interior. The following lemma shows that $\operatorname{conv}\left(X_{\alpha}\right)$ can have at most four vertices.

Lemma 4 [2, 9] Let $P \subset \mathbb{R}^{2}$ be a convex polygon with integer vertices that has no integer points in its interior.

(i) P has at most four vertices

(ii) If P has four vertices, then at least two of its four facets are parallel.

(iii) If $P$ is not a triangle with integer points in the interior of all three facets, then there exist parallel lines $\pi x=\pi_{0}$ and $\pi x=\pi_{0}+1,\left(\pi, \pi_{0}\right) \in \mathbb{Z}^{3}$, such that $P$ is contained in the corresponding split set, i.e., $P \subseteq\left\{x \in \mathbb{R}^{2}: \pi_{0} \leq\right.$ $\left.\pi x \leq \pi_{0}+1\right\}$.

\section{Representations of integer points}

In order to characterize the geometry of the facet defining inequalities for $\operatorname{conv}\left(P_{\mathrm{I}}\right)$, we exploit properties of the set of valid inequalities for $\operatorname{conv}\left(P_{\mathrm{I}}\right)$. An inequality $\sum_{j \in N} \alpha_{j} s_{j} \geq \alpha_{0}$ is facet defining for $\operatorname{conv}\left(P_{\mathrm{I}}\right)$ if and only if $\left(\alpha, \alpha_{0}\right)$ is an extreme ray of the following polyhedral cone

$$
V\left(P_{I}\right)=\left\{\left(\alpha, \alpha_{0}\right) \in \mathbb{R}^{n+1}: \alpha_{j} \geq 0, j \in U \text { and } \sum_{j \in N} \alpha_{j} s_{j} \geq \alpha_{0}, s \in S^{v}\right\}
$$

where $S^{v}:=\left\{s \in \mathbb{R}^{n}: \exists x \in \mathbb{Z}^{2}\right.$ s.t. $(x, s)$ is a vertex of $\left.\operatorname{conv}\left(P_{\mathrm{I}}\right)\right\}$.

The set $V\left(P_{I}\right)$, also known as the polar of $\operatorname{conv}\left(P_{I}\right)$, describes the set of valid inequalities for $\operatorname{conv}\left(P_{\mathrm{I}}\right)$. Recall that we are only interested in valid inequalities in standard form, i.e., valid inequalities for $\operatorname{conv}\left(P_{\mathrm{I}}\right)$ of the form $\sum_{j \in N} \alpha_{j} s_{j} \geq 1$, where $\alpha_{j} \geq 0$ for $j \in N$. To understand these inequalities, we investigate different representations of an integer point in terms of the variables $s$. 
Definition 1 Let $\sum_{j \in N} \alpha_{j} s_{j} \geq 1$ be valid for $\operatorname{conv}\left(P_{I}\right)$ and in standard form. Suppose $\bar{x}=f+\sum_{j \in N} s_{j} r^{j}$ with $0 \leq s_{j} \leq u_{j}$ for all $j \in N$.

(a) We call $s$ a representation of $\bar{x}$.

(b) The representation $s$ of $\bar{x}$ is tight if $\sum_{j \in N} \alpha_{j} s_{j}=1$.

(c) The set $T_{\alpha}(\bar{x}):=\left\{s \in \mathbb{R}^{n}: \bar{x}=f+\sum_{j \in N} s_{j} r^{j}, \sum_{j \in N} \alpha_{j} s_{j}=1\right\}$ denotes the set of tight representations of $\bar{x}$.

(d) The representation $s$ of $\bar{x}$ induces a partitioning of $N$ into the sets $S_{0}:=\left\{j \in N: s_{j}=0\right\}, S_{u}:=\left\{j \in N: s_{j}=u_{j}\right\}, S_{\text {strict }}:=\{j \in N: 0<$ $\left.s_{j}<u_{j}\right\}$.

(e) The dimension of $s$ is the dimension of the set $\operatorname{span}\left\{r^{j}: j \in S_{\text {strict }}\right\}$.

Example 1 (continued): Consider again the set (3)

$$
\begin{aligned}
P_{I}= & \left\{(x, s) \in \mathbb{Z}^{2} \times \mathbb{R}_{+}^{4}: 0 \leq s_{e} \leq 1\right. \text { and } \\
& \left.x=\left(\begin{array}{c}
0.2 \\
0.2
\end{array}\right)+\left(\begin{array}{c}
0.7 \\
0
\end{array}\right) s_{e}+\left(\begin{array}{c}
-1 \\
1
\end{array}\right) s_{1}+\left(\begin{array}{c}
1 \\
1
\end{array}\right) s_{2}+\left(\begin{array}{c}
0 \\
-1
\end{array}\right) s_{3}\right\}
\end{aligned}
$$

and the inequality (4) given by $\frac{7}{30} s_{e}+s_{1}+\frac{5}{3} s_{2}+2 s_{3} \geq 1$.

The point $\bar{x}=(0,1)$ is on the boundary of $L_{\alpha}$ (see Fig. 1). We have that $\bar{x}$ can be written in any of the following forms

$$
\begin{aligned}
& \bar{x}=f \quad+0.5 r^{1}+0.3 r^{2}, \\
& \bar{x}=f+\frac{2}{7} r^{e}+0.6 r^{1}+0.2 r^{2}, \\
& \bar{x}=f+r^{e}+0.9 r^{1} \quad+0.1 r^{3} .
\end{aligned}
$$

The representations $s^{1}=(0,0.5,0.3,0)$ and $s^{2}=\left(\frac{2}{7}, 0.6,0.2,0\right)$ give tight representations of $\bar{x}$ with respect to inequality (4) whereas the representation $s^{3}=(1,0.9,0,0.1)$ does not. The partitionings of $N$ induced by the representations $s^{2}$ and $s^{3}$ are given by the sets $S_{\mathrm{u}}^{2}=\emptyset, S_{\text {strict }}^{2}=\{e, 1,2\}, S_{0}^{2}=\{3\}$, $S_{\mathrm{u}}^{3}=\{e\}, S_{\mathrm{strict}}^{3}=\{1,3\}$, and $S_{0}^{3}=\{2\}$.

The following concept will be key in the following.

Definition $2 A$ subset $J \subseteq N$ has the linear dependence property with respect to $\alpha \in \mathbb{R}^{n}$ if for all $\lambda \in \mathbb{R}^{|J|}$,

$$
\sum_{j \in J} \lambda_{j} r^{j}=0 \Rightarrow \sum_{j \in J} \lambda_{j} \alpha_{j}=0 .
$$

The linear dependence property means, geometrically, that the boundary of $L_{\alpha}$ follows a straight line through the cone formed by the rays in $J$. 
Example 1 (continued) Consider again the set (3) and the valid inequality (4). Observe that $r^{2}=\frac{20}{7} r^{e}+r^{1}$, where we have

$$
r^{e}=\left(\begin{array}{c}
0.7 \\
0
\end{array}\right), r^{1}=\left(\begin{array}{c}
-1 \\
1
\end{array}\right) \text { and } r^{2}=\left(\begin{array}{l}
1 \\
1
\end{array}\right) .
$$

Furthermore $\alpha_{2}=\frac{20}{7} \alpha_{e}+\alpha_{1}$ since $\alpha_{e}=\frac{7}{30}, \alpha_{1}=1, \alpha_{2}=\frac{5}{3}$. Hence $\{e, 1,2\}$ satisfies the linear dependence property wrt. $\alpha$. Observe that in Fig. 1, the border of $L_{\alpha}$ follows a straight line through the cone spanned by $r^{e}$ and $r^{1}$.

Lemma 5 Let $\sum_{j \in N} \alpha_{j} s_{j} \geq 1$ be a facet defining inequality for $\operatorname{conv}\left(P_{I}\right)$ in standard form. Also let $s$ be a tight representation of $x \in \mathbb{Z}^{2}$. Then $S_{\text {strict }}$ satisfies the linear dependence property wrt. $\alpha$.

Proof: Since $s$ is a tight representation of $x$, we have $x=f+\sum_{i \in N} r^{i} s_{i}$, and

$$
\sum_{i \in N} \alpha_{i} s_{i}=1
$$

Consider multipliers $\sigma_{k}$ for $k \in S_{\text {strict }}$ (not all zero) such that $\sum_{k \in S_{\text {strict }}} \sigma_{k} r^{k}=$ 0 . There exists $\epsilon>0$ such that we have the following representations of $x$

$$
\begin{aligned}
& x=f+\sum_{i \in N \backslash S_{\text {strict }}} r^{i} s_{i}+\sum_{k \in S_{\text {strict }}} r^{k}\left(s_{k}+\sigma_{k} \epsilon\right), \\
& x=f+\sum_{i \in N \backslash S_{\text {strict }}} r^{i} s_{i}+\sum_{k \in S_{\text {strict }}} r^{k}\left(s_{k}-\sigma_{k} \epsilon\right) .
\end{aligned}
$$

We therefore have

$$
\begin{gathered}
\sum_{i \in N \backslash S_{\text {strict }}} \alpha_{i} s_{i}+\sum_{k \in S_{\text {strict }}} \alpha_{k}\left(s_{k}+\sigma_{k} \epsilon\right) \geq 1, \\
\sum_{i \in N \backslash S_{\text {strict }}} \alpha_{i} s_{i}+\sum_{k \in S_{\text {strict }}} \alpha_{k}\left(s_{k}-\sigma_{k} \epsilon\right) \geq 1 .
\end{gathered}
$$

Using (8) and (9) we obtain $\sum_{k \in S_{\text {strict }}} \sigma_{k} \alpha_{k} \epsilon \geq 0$, and using (8) and (10) we obtain $-\sum_{k \in S_{\text {strict }}} \sigma_{k} \alpha_{k} \epsilon \geq 0$. It follows that $\sum_{k \in S_{\text {strict }}} \sigma_{k} \alpha_{k}=0$.

The linear dependence property can be used to create additional tight representations from a given tight representation. Specifically, given a valid inequality $\sum_{j \in N} \alpha_{j} s_{j} \geq 1$ for $\operatorname{conv}\left(P_{\mathrm{I}}\right)$ that is in standard form, if $J \subseteq N$ has the linear dependence property wrt. $\alpha$, if $\left\{\lambda_{j}\right\}_{j \in J}$ satisfies $\sum_{j \in J} \lambda_{j} r^{j}=0$ and if $s \in \mathbb{R}_{+}^{n}$ is a tight representation of some $x \in X_{\alpha}$ that satisfies $0 \leq s_{j}+\lambda_{j} \leq u_{j}$ for all $j \in J$, then $t \in \mathbb{R}_{+}^{n}$ is also a tight representation of $x$, where $t_{j}:=s_{j}$ for $j \in N \backslash J$ and $t_{j}:=s_{j}+\lambda_{j}$ for $j \in J$. For example, in Fig. 2, the set $\{1,2,3,4\}$ satisfies the linear dependence property wrt. $\alpha$. Any representation of $x$ that use rays from $\{1,2,3,4\}$ is tight. We formalize this construction in the following lemma. 


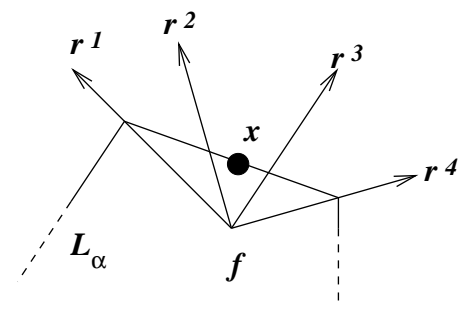

Figure 2: Example of rays that satisfy the linear dependence property

Lemma 6 Let $\sum_{j \in N} \alpha_{j} s_{j} \geq 1$ be a valid inequality for $\operatorname{conv}\left(P_{I}\right)$ that is in standard form. Suppose $s$ is a tight representation of $x \in \mathbb{Z}^{2}$, and let $t$ be a representation of $x$ satisfying $S_{u} \subseteq T_{u}$ and $T_{\text {strict }} \cup\left(T_{u} \backslash S_{u}\right) \subseteq S_{\text {strict. }}$. Then

(i) $t$ is tight.

(ii) For any $\beta \in \mathbb{R}^{n}$, if

(a) $\sum_{j \in N} \beta_{j} s_{j}=1$ and

(b) $S_{\text {strict }}$ has the linear dependence property wrt. $\beta$,

then $\sum_{j \in N} \beta_{j} t_{j}=1$.

Proof: (i) Since $s$ and $t$ are representations of $x$ we have $\sum_{j \in N} s_{j} r^{j}=\sum_{j \in N} t_{j} r^{j}$. We can rewrite this as

$$
\begin{aligned}
\sum_{j \in S_{\text {strict }}} s_{j} r^{j}+\sum_{j \in S_{\mathrm{u}}} u_{j} r^{j} & =\sum_{j \in T_{\text {strict }}} t_{j} r^{j}+\sum_{j \in T_{\mathrm{u}}} u_{j} r^{j} \\
& =\sum_{j \in T_{\text {strict }} \cup\left(T_{\mathrm{u}} \backslash S_{\mathrm{u}}\right)} t_{j} r^{j}+\sum_{j \in S_{\mathrm{u}}} u_{j} r^{j} .
\end{aligned}
$$

This implies $\sum_{j \in S_{\text {strict }}} s_{j} r^{j}=\sum_{j \in T_{\text {strict }} \cup\left(T_{\mathrm{u}} \backslash S_{\mathrm{u}}\right)} t_{j} r^{j}$. Since $T_{\text {strict }} \cup\left(T_{\mathrm{u}} \backslash S_{\mathrm{u}}\right) \subseteq$ $S_{\text {strict }}$, the linear dependence property of $S_{\text {strict }}$ wrt. $\alpha$ implies $\sum_{j \in S_{\text {strict }}} \alpha_{j} s_{j}=$ $\sum_{j \in T_{\text {strict }} \cup\left(T_{\mathrm{u}} \backslash S_{\mathrm{u}}\right)} \alpha_{j} t_{j}$. Since $s$ is a tight representation, $t$ is tight as well.

(ii) We need to prove that (a) and (b) imply $\sum_{j \in N} \beta_{j} t_{j}=1$. From what was shown in (i), we have $\sum_{j \in S_{\text {strict }}} s_{j} r^{j}=\sum_{j \in T_{\text {strict }} \cup\left(T_{\mathrm{u}} \backslash S_{\mathrm{u}}\right)} t_{j} r^{j}$. By using (b), it follows that $\sum_{j \in S_{\text {strict }}} \beta_{j} s_{j}=\sum_{j \in T_{\text {strict }} \cup\left(T_{\mathrm{u}} \backslash S_{\mathrm{u}}\right)} \beta_{j} t_{j}$. From (a) we now conclude that $\sum_{j \in N} \beta_{j} t_{j}=1$.

Example 1 (continued) Consider again the set $P_{I}$ from (3) and the valid inequality (4). We have seen earlier that $\{e, 1,2\}$ satisfies the linear dependence property wrt. $\alpha$. We also have representations $s^{1}$ and $s^{2}$ of $\bar{x}=(0,1)$ given by (6) and (7) that use rays from $\{e, 1,2\}$. Since $s^{1}$ is tight, it follows from Lemma 6 that $s^{2}$ is also tight. 
Given an integer point $x \in \mathbb{Z}^{2}$ and a tight representation $s \in T_{\alpha}(x)$ of $x$, the set $S_{\text {strict }} \cup S_{\mathrm{u}}$ denotes the relevant rays in this representation of $x$. An important question is whether the cone $\operatorname{cone}\left\{r^{j}: j \in S_{\text {strict }} \cup S_{\mathrm{u}}\right\}$ obtained from these rays cover $\mathbb{R}^{2}$ or not, since this shows whether two or three rays are needed to describe cone $\left\{r^{j}: j \in S_{\text {strict }} \cup S_{\mathrm{u}}\right\}$.

Lemma 7 Let $\sum_{j \in N} \alpha_{j} s_{j} \geq 1$ be a facet defining inequality for $\operatorname{conv}\left(P_{I}\right)$ that is in standard form. Also let $s \in \mathbb{R}^{n}$ be a tight representation of $x \in \mathbb{Z}^{2}$. Then cone $\left\{r^{j}: j \in S_{\text {strict }} \cup S_{u}\right\} \neq \mathbb{R}^{2}$.

Proof: If cone $\left\{r^{j}: j \in S_{\text {strict }} \cup S_{\mathrm{u}}\right\}=\mathbb{R}^{2}$, there exists $\sigma_{j}>0$ for $j \in S_{\text {strict }} \cup S_{\mathrm{u}}$ such that $\sum_{j \in S_{\text {strict }} \cup S_{\mathrm{u}}} \sigma_{j} r^{j}=0$. This implies there exists $\epsilon>0$ such that we have the following representation of $x$

$$
x=f+\sum_{j \in S_{\text {strict }} \cup S_{\mathrm{u}}}\left(s_{j}-\epsilon \sigma_{j}\right) r^{j} .
$$

Hence $\sum_{j \in S_{\text {strict }} \cup S_{\mathrm{u}}} \alpha_{j}\left(s_{j}-\epsilon \sigma_{j}\right) \geq 1$, and therefore $\sum_{j \in S_{\text {strict }} \cup S_{\mathrm{u}}} \sigma_{j} \alpha_{j} \leq 0$. However, since $\alpha_{j} \geq 0$ and $\sigma_{j}>0$ for $j \in S_{\text {strict }} \cup S_{\mathrm{u}}$, this means $\alpha_{j}=0$ for $j \in S_{\text {strict }} \cup S_{\mathrm{u}}$, and this contradicts that $s$ is a tight representation of $x$.

For any $x \in \mathbb{Z}^{2}$ and valid inequality $\sum_{i \in N} \alpha_{i} s_{i} \geq 1$ for $\operatorname{conv}\left(P_{\mathrm{I}}\right)$ in standard form, $T_{\alpha}(x)$ is a polyhedron. If $T_{\alpha}(x)$ is not full dimensional, there exists $\mathcal{S}_{\mathrm{u}}^{\alpha}(x) \subseteq B$ and $\mathcal{S}_{0}^{\alpha}(x) \subseteq N$ s.t. $s_{j}=u_{j}$ and $s_{k}=0$ for all $s \in T_{\alpha}(x), j \in \mathcal{S}_{\mathrm{u}}^{\alpha}(x)$ and $k \in \mathcal{S}_{0}^{\alpha}(x)$. Furthermore, $T_{\alpha}(x)$ has an inner point, i.e., a point $\bar{s} \in T_{\alpha}(x)$ that satisfies $0<\bar{s}_{j}<u_{j}$ for all $j \in N \backslash\left(\mathcal{S}_{0}^{\alpha}(x) \cup \mathcal{S}_{\mathrm{u}}^{\alpha}(x)\right)$.

Definition 3 Let $\sum_{i \in N} \alpha_{i} s_{i} \geq 1$ be valid for $\operatorname{conv}\left(P_{I}\right)$ and in standard form, and let $x \in \mathbb{Z}^{2}$ satisfy $T_{\alpha}(x) \neq \emptyset$. Define the sets

$$
\mathcal{S}_{0}^{\alpha}(x):=\bigcap_{s \in T_{\alpha}(x)} S_{0}, \quad \mathcal{S}_{\text {strict }}^{\alpha}(x):=\bigcup_{s \in T_{\alpha}(x)} S_{\text {strict }} \text { and } \mathcal{S}_{u}^{\alpha}(x):=\bigcap_{s \in T_{\alpha}(x)} S_{u}
$$

of coordinates on lower bound in all tight representations of $x$, coordinates between bounds in some tight representation of $x$, and coordinates on upper bound in all tight representations of $x$ respectively.

In the following, $s^{*} \in T_{\alpha}(x)$ denotes a representation of $x$ satisfying

$$
S_{0}^{*}=\mathcal{S}_{0}^{\alpha}(x), \quad S_{\text {strict }}^{*}=\mathcal{S}_{\text {strict }}^{\alpha}(x) \quad \text { and } \quad S_{\mathrm{u}}^{*}=\mathcal{S}_{\mathrm{u}}^{\alpha}(x) .
$$

The coefficients $\alpha \in \mathbb{R}^{n}$ in a facet defining inequality $\sum_{i \in N} \alpha_{i} s_{i} \geq 1$ for $\operatorname{conv}\left(P_{\mathrm{I}}\right)$ in standard form is the unique solution to following equality system in variables $\beta$.

$$
\sum_{j \in N} \beta_{j} s_{j}=1 \quad \text { for all } x \in X_{\alpha} \text { and } s \in T_{\alpha}(x)
$$

It is clear that the system (12) contains many redundant equalities. In the remainder of this section, we construct a system equivalent to (12) that is significantly smaller. 
Lemma 8 Let $\sum_{i \in N} \alpha_{i} s_{i} \geq 1$ be valid for $\operatorname{conv}\left(P_{I}\right)$ and in standard form. Suppose $\left\{x^{k}\right\}_{k=1}^{3} \subset \mathbb{Z}^{2}$ satisfy $x^{3}=\lambda x^{1}+(1-\lambda) x^{2}$, where $x^{1} \neq x^{2}$ and $\left.\lambda \in\right] 0,1[$. If there exists a tight $2 D$ representation (see Definition 1.(e)) of either $x^{1}$ or $x^{2}$, then the following equalities in variables $\beta$

$$
\sum_{j \in N} \beta_{j} s_{j}=1 \quad \text { for all } s \in T_{\alpha}\left(x^{3}\right)
$$

are implied by the following equalities in variables $\beta$

$$
\sum_{j \in N} \beta_{j} s_{j}=1 \quad \text { for all } s \in T_{\alpha}\left(x^{1}\right) \cup T_{\alpha}\left(x^{2}\right) .
$$

Proof: Suppose $\left\{x^{k}\right\}_{k=1}^{3} \subset \mathbb{Z}^{2}$ satisfy $x^{3}=\lambda x^{1}+(1-\lambda) x^{2}$, where $x^{1} \neq x^{2}$ and $\lambda \in] 0,1\left[\right.$. Let $\bar{\beta} \in \mathbb{R}^{n}$ satisfy (14), and let $\bar{t} \in T_{\alpha}\left(x^{3}\right)$ be an arbitrary tight representation of $x^{3}$. We will show that $\sum_{j \in N} \bar{\beta}_{j} \bar{t}_{j}=1$.

Let $s^{*}$ and $t^{*}$ be tight representations of $x^{1}$ and $x^{2}$ that satisfy (11) for $x^{1}$ and $x^{2}$ respectively. Observe that $\bar{s}:=\lambda s^{*}+(1-\lambda) t^{*}$ is a tight representation of $x^{3}$ satisfying

$$
\bar{S}_{0}=\bigcap_{s \in T_{\alpha}\left(x^{1}\right) \cup T_{\alpha}\left(x^{2}\right)} S_{0} \quad \text { and } \quad \bar{S}_{u}=\bigcap_{s \in T_{\alpha}\left(x^{1}\right) \cup T_{\alpha}\left(x^{2}\right)} S_{u} .
$$

In other words, the representation $\bar{s}$ of $x^{3}$ only has a coordinate which is on lower bound (on upper bound) if all representations of $x^{1}$ and $x^{2}$ are on lower bound (on upper bound) on this coordinate.

To finish the proof we show that the representations $\bar{s}$ and $\bar{t}$ of $x^{3}$ satisfy

(i) $\bar{S}_{\text {strict }}$ satisfies the linear dependence property wrt. $\bar{\beta}$.

(ii) $\bar{S}_{\mathrm{u}} \subseteq \bar{T}_{\mathrm{u}}$ and

(iii) $\bar{T}_{\text {strict }} \cup\left(\bar{T}_{\mathrm{u}} \backslash \bar{S}_{\mathrm{u}}\right) \subseteq \bar{S}_{\text {strict }}$

Lemma 6.(ii) then shows $\sum_{j \in N} \bar{\beta}_{j} \bar{t}_{j}=1$ which proves the lemma.

By assumption either $x^{1}$ or $x^{2}$ have a tight $2 \mathrm{D}$ representation. Without loss of generality suppose $x^{1}$ has a tight $2 \mathrm{D}$ representation. Let $s^{1}$ be a tight $2 \mathrm{D}$ representation of $x^{1}$, and let $s^{2}$ be an arbitrary tight representation of $x^{2}$

$$
x^{1}=f+\sum_{j \in N} s_{j}^{1} r^{j} \text { and } x^{2}=f+\sum_{j \in N} s_{j}^{2} r^{j} .
$$

Define the tight representation $\bar{z}:=\frac{\lambda}{2} s^{1}+\frac{1-\lambda}{2} s^{2}+\frac{1}{2} \bar{t}$ of $x^{3}$. Observe that, if $j \in \bar{S}_{0}$, then (15) shows $s_{j}=0$ for every representation $s \in T_{\alpha}\left(x^{1}\right) \cup T_{\alpha}\left(x^{2}\right)$, and therefore $s_{j}^{1}=s_{j}^{2}=0$. Similarly if $j \in \bar{S}_{\mathrm{u}}$, then $s_{j}^{1}=s_{j}^{2}=u_{j}$.

Since $s^{1}$ is a $2 \mathrm{D}$ representation of $x^{1}$, there exists linearly independent vectors $r^{l_{1}}$ and $r^{l_{2}}$ with $l_{1}, l_{2} \in S_{\text {strict }}^{1}$. Therefore, for every $j \in N$, there exists $\sigma_{l_{1}}^{j}, \sigma_{l_{2}}^{j} \in$ $\mathbb{R}$ such that $r^{j}=\sigma_{l_{1}}^{j} r^{l_{1}}+\sigma_{l_{2}}^{j} r^{l_{2}}$. We can now prove (i)-(iii). 
(i) Let $\lambda^{\prime} \in \mathbb{R}^{n}$ satisfy $\sum_{j \in \bar{S}_{\text {strict }}} \lambda_{j}^{\prime} r^{j}=0$. Since $\bar{s}=\lambda s^{*}+(1-\lambda) t^{*}$ with $s^{*} \in T_{\alpha}\left(x^{1}\right)$ and $t^{*} \in T_{\alpha}\left(x^{2}\right)$, and $\bar{\beta}$ satisfies (14), we have $\sum_{j \in N} \bar{\beta}_{j} s_{j}^{*}=1$ and $\sum_{j \in N} \bar{\beta}_{j} t_{j}^{*}=1$. Now partition the set $\left\{j \in \bar{S}_{\text {strict }}: \lambda_{j}^{\prime} \neq 0\right\}$ into the sets $S^{+}:=\left\{j \in \bar{S}_{\text {strict }}: \lambda_{j}^{\prime}>0, s_{j}^{*}<u_{j}\right\}, S^{-}:=\left\{j \in \bar{S}_{\text {strict }}: \lambda_{j}^{\prime}<0, s_{j}^{*}>0\right\}$, $T^{+}:=\left\{j \in \bar{S}_{\text {strict }}: \lambda_{j}^{\prime}>0, j \notin S^{+}\right\}$and $T^{-}:=\left\{j \in \bar{S}_{\text {strict }}: \lambda_{j}^{\prime}<0, j \notin S^{-}\right\}$. This implies there exists $\epsilon>0$ s.t. $x^{1}=f+\sum_{j \in N} \bar{s}_{j}^{*} r^{j}+\epsilon \sum_{j \in S^{+} \cup S^{-}} \lambda_{j}^{\prime} r^{j}$ and $x^{2}=f+\sum_{j \in N} \bar{t}_{j}^{*} r^{j}+\epsilon \sum_{j \in T^{+} \cup T^{-}} \lambda_{j}^{\prime} r^{j}$ are tight representations of $x^{1}$ and $x^{2}$ respectively. Hence from (14) we have $\sum_{j \in N} \bar{\beta}_{j} \bar{s}_{j}^{*}+\epsilon \sum_{j \in S^{+} \cup S^{-}} \bar{\beta}_{j} \lambda_{j}^{\prime}=1$ and $\sum_{j \in N} \bar{\beta}_{j} \bar{t}_{j}^{*}+\epsilon \sum_{j \in T^{+} \cup T^{-}} \bar{\beta}_{j} \lambda_{j}^{\prime}=1$, which implies $\sum_{j \in \bar{S}_{\text {strict }}} \bar{\beta} \lambda_{j}^{\prime}=0$.

(ii) Suppose, for a contradiction, that $\bar{j} \in \bar{S}_{\mathrm{u}}$ and $\bar{j} \notin \bar{T}_{\mathrm{u}}$. Since $\bar{j} \in \bar{S}_{\mathrm{u}}$, (15) shows $s_{\bar{j}}=u_{\bar{j}}$ for all tight representations $s \in T_{\alpha}\left(x^{1}\right) \cup T_{\alpha}\left(x^{2}\right)$ of $x^{1}$ and $x^{2}$. Hence $\bar{j} \in S_{\mathrm{u}}^{1} \cap S_{\mathrm{u}}^{2}$, and therefore $\bar{j} \notin \bar{T}_{\mathrm{u}}$ implies $\bar{j} \in \bar{Z}_{\text {strict. }}$. We have $\bar{j} \neq l_{1}$ and $\bar{j} \neq l_{2}$ since $\bar{j} \notin S_{\text {strict }}^{1}$ and $l_{1}, l_{2} \in S_{\text {strict. }}^{1}$. Furthermore, since $\left\{l_{1}, l_{2}, \bar{j}\right\} \subseteq \bar{Z}_{\text {strict }},\left\{l_{1}, l_{2}, \bar{j}\right\}$ satisfies the linear dependence property wrt. $\alpha$. Hence there exists $\epsilon>0$ such that $x^{1}=f+\sum_{j \in N} s_{j}^{1} r^{j}+\epsilon\left(\sigma_{l_{1}}^{\bar{j}} r^{l_{1}}+\sigma_{l_{2}}^{\bar{j}} r^{l_{2}}-r^{\bar{j}}\right)$ is a tight representation of $x^{1}$. However, this contradicts that $s_{\bar{j}}=u_{\bar{j}}$ for all tight representations $s$ of $x^{1}$.

(iii) Observe that (ii) implies $\bar{T}_{\text {strict }} \cap \bar{S}_{\mathrm{u}}=\emptyset:$ If $\bar{j} \in \bar{S}_{\mathrm{u}}$, then (ii) implies $\bar{j} \in \bar{T}_{\mathrm{u}}$, and therefore $\bar{j} \notin \bar{T}_{\text {strict }}$. Hence, to show (iii), it suffices to show $\bar{T}_{\mathrm{u}} \cup \bar{T}_{\text {strict }} \subseteq \bar{S}_{\mathrm{u}} \cup \bar{S}_{\text {strict. }}$. Suppose for a contradiction that $\bar{j} \in \bar{T}_{\mathrm{u}} \cup \bar{T}_{\text {strict }}$ and $\bar{j} \in \bar{S}_{0}$. Since $\bar{j} \in \bar{S}_{0}$, (15) shows that $s_{\bar{j}}=0$ for every tight representation $s \in T_{\alpha}\left(x^{1}\right) \cup T_{\alpha}\left(x^{2}\right)$ of $x^{1}$ and $x^{2}$. We therefore have $s \frac{1}{\bar{j}}=s_{\bar{j}}^{2}=0$. Since $\bar{j} \in$ $\bar{T}_{\mathrm{u}} \cup \bar{T}_{\text {strict }}$, this implies $\bar{j} \in \bar{Z}_{\text {strict }}$. Furthermore we have $\bar{j} \neq l_{1}$ and $\bar{j} \neq l_{2}$ since $\bar{j} \notin S_{\text {strict }}^{1}$ and $l_{1}, l_{2} \in S_{\text {strict }}^{1}$. Since $\left\{l_{1}, l_{2}, \bar{j}\right\} \subseteq \bar{Z}_{\text {strict }}$, Lemma 5 shows $\left\{l_{1}, l_{2}, \bar{j}\right\}$ satisfies the linear dependence property wrt. $\alpha$. Hence there exists $\epsilon>0$ such that $x^{1}=f+\sum_{i \in N} s_{i}^{1} r^{i}+\epsilon\left(r^{\bar{j}}-\sigma_{l_{1}}^{\bar{j}} r^{l_{1}}-\sigma_{l_{2}}^{\bar{j}} r^{l_{2}}\right)$ is a tight representation of $x^{1}$. However, this contradicts that $s_{\bar{j}}=0$ for all tight representations $s$ of $x^{1}$.

We now identify "important rays".

Definition 4 Given $x^{i} \in X_{\alpha} \cap \mathbb{Z}^{2}$, define

(i) $I^{U}\left(x^{i}\right)$ to be s.t. cone $\left(\left\{r^{j}\right\}_{j \in I^{U}\left(x^{i}\right)}\right)=\operatorname{cone}\left(\left\{r^{j}\right\}_{j \in \mathcal{S}_{\text {strict }}^{\alpha}\left(x^{i}\right) \cap U}\right)$,

(ii) $I\left(x^{i}\right)$ to be $I^{U}\left(x^{i}\right)$ if $\left|I^{U}\left(x^{i}\right)\right|=2$. Otherwise $I\left(x^{i}\right)$ is defined to be s.t. cone $\left(\left\{r^{j}\right\}_{j \in I\left(x^{i}\right)}\right)=\operatorname{cone}\left(\left\{r^{j}\right\}_{j \in \mathcal{S}_{\text {strict }}^{\alpha}\left(x^{i}\right)}\right)$.

Observe that Lemma 7 implies that the cardinality of these two sets is at most 2. Using this notation, we now reformulate system (12) as follows.

Lemma 9 Let $\sum_{i \in N} \alpha_{i} s_{i} \geq 1$ be valid for $\operatorname{conv}\left(P_{I}\right)$ and in standard form, let $\bar{x} \in \mathbb{Z}^{2}$, and suppose $\bar{x}$ has a tight $2 D$ representation. Also, given $j \in$ $\mathcal{S}_{\text {strict }}^{\alpha}(\bar{x}) \cap U$ and $k \in I^{U}(\bar{x})$, let $\sigma_{k}^{j} \geq 0$ be defined such that $r^{j}=\sum_{k \in I^{U}(\bar{x})} \sigma_{k}^{j} r^{k}$. Finally, given $j \in \mathcal{S}_{\text {strict }}^{\alpha}(\bar{x}) \cap B$ and $k \in I(\bar{x})$, let $\sigma_{k}^{j}$ be defined such that 
$r^{j}=\sum_{k \in I(\bar{x})} \sigma_{k}^{j} r^{k}$. The two linear systems in unknowns $\beta$

$$
\sum_{k \in N} \beta_{k} s_{k}=1 \quad \text { for all } s \in T_{\alpha}(\bar{x})
$$

and

$$
\begin{array}{ll}
\sum_{k \in N} \beta_{k} \bar{t}_{k}=1, & \\
\beta_{j}=\sum_{k \in I^{U}(\bar{x})} \sigma_{k}^{j} \beta_{k} & \text { for all } j \in\left(\mathcal{S}_{\text {strict }}^{\alpha}(\bar{x}) \cap U\right) \backslash I^{U}(\bar{x}), \\
\beta_{j}=\sum_{k \in I(\bar{x})} \sigma_{k}^{j} \beta_{k} & \text { for all } j \in\left(\mathcal{S}_{\text {strict }}^{\alpha}(\bar{x}) \cap B\right) \backslash I(\bar{x})
\end{array}
$$

have the same solution set.

Proof: We call the solution sets of (16) and (17)-(19) for $X_{1}$ and $X_{2}$ respectively. Let $\bar{t}$ be the representation of $\bar{x}$ that satisfies (11).

We first prove $X_{1} \subseteq X_{2}$. Therefore suppose $\beta \in X_{1}$. We prove that (18) holds. The proof for (19) is similar. Consider $l \in\left(\mathcal{S}_{\text {strict }}^{\alpha}(\bar{x}) \cap U\right) \backslash I^{U}(\bar{x})$. Observe that, since $I^{U}(\bar{x}) \subseteq \mathcal{S}_{\text {strict }}^{\alpha}(\bar{x})$, we have $I^{U}(\bar{x}) \cup\{l\} \subseteq \bar{T}_{\text {strict }}$ and therefore Lemma 5 shows that $I^{U}(\bar{x}) \cup\{l\}$ satisfies the linear dependence property wrt. $\alpha$. Hence $\alpha_{l}=\sum_{i \in I^{U}(\bar{x})} \alpha_{i} \sigma_{i}^{l}$. We need to show $\beta_{l}=\sum_{i \in I^{U}(\bar{x})} \beta_{i} \sigma_{i}^{l}$. Since $I^{U}(\bar{x}) \cup\{l\}$ satisfies the linear dependence property wrt. $\alpha$, there exists $\epsilon>0$ s.t. the representation $\bar{x}=f+\sum_{j \in N} \bar{t}_{j} r^{j}+\epsilon\left(r^{l}-\sum_{i \in I^{U}(\bar{x})} \sigma_{i}^{l} r^{i}\right)$ of $\bar{x}$ is tight. Since there is an equality of (16) for every tight representation of $\bar{x}$, we have $\sum_{j \in N} \beta_{j} \bar{t}_{j}+\epsilon\left(\beta_{l}-\sum_{i \in I^{U}(\bar{x})} \beta_{i} \sigma_{i}^{l}\right)=1$. Therefore, since $\sum_{j \in N} \beta_{j} \bar{t}_{j}=1$, we have $\beta_{l}-\sum_{i \in I^{U}(\bar{x})} \beta_{i} \sigma_{i}^{l}=0$ which shows $\beta \in X_{2}$.

We now prove that $X_{2} \subseteq X_{1}$. Let $\beta \in X_{2}$ and $\bar{w} \in T_{\alpha}(\bar{x})$ be arbitrary. We must prove $\sum_{l \in N} \beta_{l} \bar{w}_{l}=1$. Define

$$
X^{u}:=\bar{T}_{\mathrm{u}} \cap \bar{W}_{\mathrm{u}}, \quad Y:=\left(\bar{T}_{u} \backslash X^{u}\right) \cup \bar{T}_{\text {strict }} \quad Z:=\left(\bar{W}_{u} \backslash X^{u}\right) \cup \bar{W}_{\text {strict }} .
$$

Observe that $X^{u}$ and $Y$ form a partitioning of $\bar{T}_{\text {strict }} \cup \bar{T}_{\mathrm{u}}$, and that $X^{u}$ and $Z$ form a partitioning of $\bar{W}_{\text {strict }} \cup \bar{W}_{\mathrm{u}}$. Also observe that, since $\bar{T}_{\mathrm{u}}=\bigcap_{s \in T_{\alpha}(\bar{x})} S_{\mathrm{u}}$, and $\bar{w} \in T_{\alpha}(\bar{x})$, we have $X^{u}=\bar{T}_{\mathrm{u}}$ and $Y=\bar{T}_{\text {strict }}$. We may write

$$
\begin{aligned}
& \bar{x}=f+\sum_{i \in Y} \bar{t}_{i} r^{i}+\sum_{j \in X^{u}} u_{j} r^{j} \quad \text { and } \\
& \bar{x}=f+\sum_{i \in Z} \bar{w}_{i} r^{i}+\sum_{j \in X^{u}} u_{j} r^{j} .
\end{aligned}
$$

Both $\bar{t}$ and $\bar{w}$ are tight representations of $\bar{x}$ wrt. $\sum_{i \in N} \alpha_{i} s_{i} \geq 1$. Therefore, since $\mathcal{S}_{\text {strict }}^{\alpha}(\bar{x})$ consists of those coordinates that are between bounds in some tight representation of $\bar{x}$ wrt. $\sum_{i \in N} \alpha_{i} s_{i} \geq 1$, we have $\bar{T}_{\text {strict }} \cup \bar{W}_{\text {strict }} \subseteq \mathcal{S}_{\text {strict }}^{\alpha}(\bar{x})$. Also, for $l \in \bar{W}_{u} \backslash X^{u}$, we have $0<\bar{t}_{l}<u_{l}$ and $\bar{w}_{l}=u_{l}$, and therefore the 
tight representation $\frac{1}{2} \bar{t}+\frac{1}{2} \bar{w}$ of $\bar{x}$ satisfies $0<\frac{1}{2} \bar{l}_{l}+\frac{1}{2} \bar{w}_{l}<u_{l}$. It follows that $\bar{W}_{u} \backslash X^{u} \subseteq \mathcal{S}_{\text {strict }}^{\alpha}(\bar{x})$, and therefore $Z \subseteq \mathcal{S}_{\text {strict }}^{\alpha}(\bar{x})$. Assume that $I(\bar{x})=I^{U}(\bar{x})$ (otherwise the proof applies with $I(\bar{x})$ ). From (18) it follows that

$$
\beta_{l}=\sum_{i \in I^{U}(\bar{x})} \sigma_{i}^{l} \beta_{i} \quad l \in(Y \cup Z) \backslash I^{U}(\bar{x}) .
$$

For $i \in I^{U}(\bar{x})$, define

$$
\bar{t}_{Y}^{i}:=\bar{t}_{i}+\sum_{l \in Y \backslash I^{U}(\bar{x})} \sigma_{i}^{l} \bar{t}_{l} \quad \text { and } \quad \bar{w}_{Z}^{i}:=\bar{w}_{i}+\sum_{l \in Z \backslash I^{U}(\bar{x})} \sigma_{i}^{l} \bar{w}_{l} .
$$

Adding $\sum_{l \in Y \backslash I^{U}(\bar{x})} \bar{t}_{l}\left(-r^{l}+\sum_{i \in I^{U}(\bar{x})} \sigma_{i}^{l} r^{i}\right)(=0)$ to the right-hand-side of (20), and adding $\sum_{l \in Z \backslash I^{U}(\bar{x})} \bar{w}_{l}\left(-r^{l}+\sum_{i \in I^{U}(\bar{x})} \sigma_{i}^{l} r^{i}\right)(=0)$ to the right-hand-side of (21), we obtain

$$
\begin{aligned}
& \bar{x}=f+\sum_{i \in I^{U}(\bar{x})} \bar{t}_{Y}^{i} r^{i}+\sum_{i \in X^{u}} u_{i} r^{i} \\
& \bar{x}=f+\sum_{i \in I^{U}(\bar{x}) \cap Z} \bar{w}_{Z}^{i} r^{i}+\sum_{i \in I^{U}(\bar{x}) \backslash Z}\left(\bar{w}_{Z}^{i}-\bar{w}_{i}\right) r^{i}+\sum_{i \in X^{u}} u_{i} r^{i}
\end{aligned}
$$

Both (23) and (24) give an expression for the vector $\bar{x}-f-\sum_{i \in X^{U}} u_{i} r^{i}$ as an element of cone $\left(\left\{r^{i}\right\}_{i \in I^{U}(\bar{x})}\right)$. Since the non-negative numbers involved in this expression are unique, we have $\bar{t}_{Y}^{i}=\bar{w}_{Z}^{i}$ for $i \in I^{U}(\bar{x}) \cap Z$ and $\bar{t}_{Y}^{i}=\bar{w}_{Z}^{i}-\bar{w}_{i}$ for $i \in I^{U}(\bar{x}) \backslash Z$. We may now write, denoting $I:=I^{U}(\bar{x})$,

$$
\begin{aligned}
1 & =\sum_{i \in Y} \bar{t}_{i} \beta_{i}+\sum_{i \in X^{u}} u_{i} \beta_{i} \\
& \left.=\sum_{i \in Y} \bar{t}_{i} \beta_{i}+\sum_{i \in X^{u}} u_{i} \beta_{i}+\sum_{l \in Y \backslash I} \bar{t}_{l}\left(-\beta_{l}+\sum_{i \in I} \sigma_{i}^{l} \beta_{i}\right)\right) \\
& =\sum_{i \in I} \beta_{i} \bar{t}_{Y}^{i}+\sum_{i \in X^{u}} u_{i} \beta_{i} \\
& \left.=\sum_{i \in I \cap Z} \beta_{i} \bar{w}_{Z}^{i}+\sum_{i \in I \backslash Z} \beta_{i}\left(\bar{w}_{Z}^{i}-\bar{w}_{i}\right)+\sum_{i \in X^{u}} u_{i} \beta_{i}-\sum_{l \in Z \backslash I} \bar{w}_{l}\left(-\beta_{l}+\sum_{i \in I} \sigma_{i}^{l} \beta_{i}\right)\right) \\
& =\sum_{i \in N} \bar{w}_{i} \beta_{i}+\sum_{i \in I \cap Z} \beta_{i}\left(\bar{w}_{Z}^{i}-\bar{w}_{i}\right)+\sum_{i \in I \backslash Z} \beta_{i}\left(\bar{w}_{Z}^{i}-\bar{w}_{i}\right)-\sum_{i \in I} \beta_{i}\left(\bar{w}_{Z}^{i}-\bar{w}_{i}\right) \\
& =\sum_{i \in N} \bar{w}_{i} \beta_{i} .
\end{aligned}
$$

The next step is to consider possible interactions between the sets $I^{U}\left(x^{i}\right)$ and $I\left(x^{i}\right)$ for different vertices of $\operatorname{conv}\left(X_{\alpha}\right)$. 
Observation 2 Let $\sum_{j \in N} \alpha_{j} s_{j} \geq 1$ be facet defining for $\operatorname{conv}\left(P_{I}\right)$ and in standard form. If $\mathcal{S}_{\text {strict }}^{\alpha}\left(x^{2}\right) \subseteq \mathcal{S}_{\text {strict }}^{\alpha}\left(x^{1}\right)$, then the equalities

$$
\beta_{j}=\sum_{i \in I\left(x^{2}\right)} \sigma_{i}^{j} \beta_{i} \quad \text { for all } j \in\left(\mathcal{S}_{\text {strict }}^{\alpha}\left(x^{2}\right) \cap B\right) \backslash I\left(x^{2}\right),
$$

are implied by the equalities

$$
\beta_{j}=\sum_{i \in I\left(x^{1}\right)} \sigma_{i}^{j} \beta_{i} \quad \text { for all } j \in\left(\mathcal{S}_{\text {strict }}^{\alpha}\left(x^{1}\right) \cap B\right) \backslash I\left(x^{1}\right) .
$$

If $\left(\mathcal{S}_{\text {strict }}^{\alpha}\left(x^{2}\right) \cap U\right) \subseteq\left(\mathcal{S}_{\text {strict }}^{\alpha}\left(x^{1}\right) \cap U\right)$, then the equalities

$$
\beta_{j}=\sum_{i \in I^{U}\left(x^{2}\right)} \sigma_{i}^{j} \beta_{i} \quad \text { for all } j \in\left(\mathcal{S}_{\text {strict }}^{\alpha}\left(x^{2}\right) \cap U\right) \backslash I^{U}\left(x^{2}\right),
$$

are implied by the equalities

$$
\beta_{j}=\sum_{i \in I^{U}\left(x^{1}\right)} \sigma_{i}^{j} \beta_{i} \quad \text { for all } j \in\left(\mathcal{S}_{\text {strict }}^{\alpha}\left(x^{1}\right) \cap U\right) \backslash I^{U}\left(x^{1}\right) .
$$

Lemma 10 Let $\sum_{j \in N} \alpha_{j} s_{j} \geq 1$ be facet defining for $\operatorname{conv}\left(P_{I}\right)$ and in standard form. If $j \in U$ satisfies $j \in \mathcal{S}_{\text {strict }}^{\alpha}\left(x^{1}\right) \backslash I^{U}\left(x^{1}\right)$, then $j \notin I^{U}\left(x^{2}\right)$.

Proof: Assume, for a contradiction, that $j \in U, j \in \mathcal{S}_{\text {strict }}^{\alpha}\left(x^{1}\right) \backslash I^{U}\left(x^{1}\right)$ and $j \in I^{U}\left(x^{2}\right)$. Let $s^{*}$ be a tight representation of $x^{2}$ satisfying (11), and let $k, l \in I^{U}\left(x^{1}\right)$, which by definition means $k, l \in U$. Lemma 5 implies that there exist $\sigma_{k}, \sigma_{l} \geq 0$ satisfying $r^{j}=\sigma_{k} r^{k}+\sigma_{l} r^{l}$ and $\alpha_{j}=\sigma_{k} r^{k}+\sigma_{l} \alpha_{l}$. Hence there exists $\epsilon>0$ such that $x^{2}=f+\sum_{i \in N} s_{i}^{*} r^{i}+\epsilon\left(-r^{j}+\sigma_{k} r^{k}+\sigma_{l} r^{l}\right)$ is a valid and tight representation of $x^{2}$. This implies $k, l \in \mathcal{S}_{\text {strict }}^{\alpha}\left(x^{2}\right)$, which is a contradiction since $j \in I^{U}\left(x^{2}\right)$ and $r^{j} \in \operatorname{cone}\left\{r^{k}, r^{l}\right\}$.

We can now prove the main result of this section, namely that for a facet $\sum_{j \in N} \alpha_{j} s_{j} \geq 1$ of $\operatorname{conv}\left(P_{I}\right)$ in standard form, $\alpha$ is the unique solution of a system consisting of one equation expressing the tightness of each vertex of $\operatorname{conv}\left(X_{\alpha}\right)$, at most one linear dependence property for each unbounded ray and possibly some linear dependence properties for bounded rays.

Theorem 1 Consider the set (5). Let $X^{v}$ denote the set of vertices of $\operatorname{conv}\left(X_{\alpha}\right)$. If $\alpha$ is an extreme point of (5), then $\alpha$ is the unique solution to the following system in variables $\beta$

$$
\begin{aligned}
& \sum_{j=1}^{n} \beta_{j} s_{j}^{x}=1 \quad \text { for all } x \in X^{v}, \\
& \beta_{j}=\sum_{k \in I(x)} \sigma_{j}^{k} \beta_{k} \quad \text { for all } x \in X_{\alpha} \text { and } j \in\left(\mathcal{S}_{\text {strict }}^{\alpha}(x) \cap B\right) \backslash I(x), \\
& \beta_{j}=\sum_{k \in I^{U}(x)} \sigma_{j}^{k} \beta_{k} \quad \text { for all } x \in X_{\alpha} \text { and } j \in\left(\mathcal{S}_{\text {strict }}^{\alpha}(x) \cap U\right) \backslash I^{U}(x),
\end{aligned}
$$


where $s^{x} \in T_{\alpha}(x)$ satisfies $s_{i}^{x}>0$ for all $i \in I^{U}(x)$ and $s_{j}^{x}=0$ for all $j \in$ $U \backslash I^{U}(x)$. Furthermore, the system (25)-(26) is also uniquely solvable.

Proof: Consider the set (5). An extreme point satisfies a subset of the inequalities with equality. These we call the tightness equalities. Using Lemma 8, we know that we only need to consider equations corresponding to vertices of $\operatorname{conv}\left(X_{\alpha}\right)$ - except for possibly points $x^{3} \in X_{\alpha}$ that are true convex combinations of vertices $x^{1}$ and $x^{2}$ of $\operatorname{conv}\left(X_{\alpha}\right)$ that do not have 2D representations. We will deal with the latter case later in the proof. Then using Lemma 9, we can write the system of tightness equations equivalently as a system consisting of one tightness equation (25) per vertex $x$ of $\operatorname{conv}\left(X_{\alpha}\right)$, and equations of type (26)-(27). Observe that, $\bar{s}_{i}^{x}$ may be nonzero for $i \in U \backslash I^{U}(x)$. However we can create a new tight representation (using $r^{i}=\sum_{k \in I^{U}(x)} \sigma_{i}^{k} r^{k}$ together with $\left.\alpha_{i}=\sum_{k \in I^{U}(x)} \sigma_{i}^{k} \alpha_{k}\right)$ that satisfies $s_{i}^{x}=0$ for $i \in U \backslash I^{U}(x)$.

We now classify the unbounded rays in two sets. Let

$U_{1}:=\left\{i \in U \mid\right.$ there exists a vertex $x$ of $\operatorname{conv}\left(X_{\alpha}\right)$ with $\left.i \in I^{U}(x)\right\}$,

$U_{2}:=\left\{i \in U \mid\right.$ there exists a vertex $x$ of $\operatorname{conv}\left(X_{\alpha}\right)$ with $\left.i \in \mathcal{S}_{\text {strict }}^{\alpha}(x) \backslash I^{U}(x)\right\}$.

In Lemma 10 we have proved that $U_{1} \cap U_{2}=\emptyset$. Observe that if $i \in U_{2}$, then $\beta_{i}$ only appears once in the equations (27). Deleting these equalities from (27) therefore leaves a system that remains uniquely solvable.

It remains to check the case when there exist $x^{1}, x^{2}, x^{3} \in X_{\alpha}$ and $\left.\lambda \in\right] 0,1[$ such that $x^{3}=\lambda x^{1}+(1-\lambda) x^{2}$ and neither $x^{1}$ nor $x^{2}$ admit a $2 \mathrm{D}$ representation. This implies that $x^{1}$ and $x^{2}$ admit exactly one tight representation, say $T_{\alpha}\left(x^{1}\right)=$ $\left\{s^{1}\right\}$ and $T_{\alpha}\left(x^{2}\right)=\left\{s^{2}\right\}$. Consider a tight representation $s^{3} \in T_{\alpha}\left(x^{3}\right)$ of $x^{3}$. If $S_{\text {strict }}^{3} \cup S_{u}^{3} \subseteq S_{\text {strict }}^{1} \cup S_{u}^{1} \cup S_{\text {strict }}^{2} \cup S_{u}^{2}$, the equation derived from the tightness of the representation $s^{3}$ is a convex combination of those obtained from $s^{1}$ and $s^{2}$ (see the proof of Lemma 8). Assume now there exists $J \subseteq\left(S_{\text {strict }}^{3} \cup S_{u}^{3}\right), J \cap$ $\left(S_{\text {strict }}^{1} \cup S_{u}^{1} \cup S_{\text {strict }}^{2} \cup S_{u}^{2}\right)=\emptyset$ with $|J| \geq 1$. Now consider the tight representation $t^{3}:=\frac{\lambda}{2} s^{1}+\frac{1-\lambda}{2} s^{2}+\frac{1}{2} s^{3}$ of $x^{3}$. Let $K \subseteq N$ denote those coordinates of $s^{1}, s^{2}$ and $s^{3}$ for which not all representations have identical values, i.e., $s_{i}^{1}=s_{i}^{2}=s_{i}^{3}$ for $i \notin K$. Observe that $s^{1}$ and $s^{2}$ must differ on at least two coordinates, since otherwise we would have either $\sum_{i \in N} \alpha_{i} s_{i}^{1} \neq 1$ or $\sum_{i \in N} \alpha_{i} s_{i}^{2} \neq 1$. Furthermore, since $|J| \geq 1$, we have $|K| \geq 3$. Also note that $K \subseteq T_{\text {strict }}^{3}$, and therefore $K$ satisfies the linear dependence property wrt. $\alpha$. We now consider $\tau:=$ $s^{3}-\lambda s^{1}-(1-\lambda) s^{2}$ which satisfies $0=\sum_{j \in K} \tau_{j} r^{j}$. Observe that the equation $0=\sum_{j \in K} \tau_{j} \beta_{j}$ must be a linear combination of equations of type (26)-(27). It follows that the equation $\sum_{j \in N} s_{j}^{3} \beta_{j}=1$ is the linear combination of equations of type (26)-(27) obtained by $\lambda\left(\sum_{j \in N} s_{j}^{1} \beta_{j}=1\right)+(1-\lambda)\left(\sum_{j \in N} s_{j}^{2} \beta_{j}=1\right)$.

\section{A characterization of the facets of $\operatorname{conv}\left(P_{\mathbf{I}}\right)$}

In this section we focus on the set $L_{\alpha}$. We assume $\alpha_{j}>0$ for all $j \in U$. Due to the direct correspondence between the set $L_{\alpha}$ and a facet defining inequality 
$\sum_{j \in N} \alpha_{j} s_{j} \geq 1$ for $\operatorname{conv}\left(P_{\mathrm{I}}\right)$ in standard form, this gives a characterization of the facets of $\operatorname{conv}\left(P_{\mathrm{I}}\right)$. We first provide some general results on the structure of $L_{\alpha}$ in Sect. 4.1. We then review the main results in [1] in the case where $B=\emptyset$ in Sect. 4.2. Finally, in Sect. 4.3, we characterize $L_{\alpha}$ when exactly one upper bound is present, i.e., when $|B|=1$. The presence of an upper bound might seem to be only a minor extension. However, as we will demonstrate later, the addition of an upper bound on a variable substantially complicates the geometry of $L_{\alpha}$. Indeed, whereas $L_{\alpha}$ is either a triangle or a quadrilateral when no upper bounds are present, $L_{\alpha}$ can also be a pentagon when an upper bound on a variable is present. The following theorem was proved in [1].

Theorem 2 Suppose $B=\emptyset$. Let $\sum_{j \in N} \alpha_{j} s_{j} \geq 1$ be a facet defining inequality for $\operatorname{conv}\left(P_{I}\right)$ that satisfies $\alpha_{j}>0$ for all $j \in N$. Then $L_{\alpha}$ is a polygon with at most four vertices.

Theorem 2 shows that there exists a set $S \subseteq N$ such that $|S| \leq 4$ and $\sum_{j \in S} \alpha_{j} s_{j} \geq 1$ is facet defining for $\operatorname{conv}\left(P_{\mathrm{I}}(S)\right)$, where

$$
P_{\mathrm{I}}(S):=\left\{(x, s) \in \mathbb{Z}^{2} \times \mathbb{R}_{+}^{|S|}: x=f+\sum_{j \in S} s_{j} r^{j}\right\}
$$

The main theorem in this section is the following.

Theorem 3 Suppose $|B|=1$. Let $\sum_{j \in N} \alpha_{j} s_{j} \geq 1$ be a facet defining inequality in standard form for $\operatorname{conv}\left(P_{I}\right)$ that satisfies $\alpha_{j}>0$ for all $j \in U$. Then $L_{\alpha}$ is a polygon with at most five vertices.

Throughout this section we assume that no two rays point in the same direction.

\subsection{General geometric statements about $L_{\alpha}$}

The set $L_{\alpha}$ is the projection of a polyhedron onto the 2-dimensional plane. It is therefore a polygon. First we characterize all points that are candidates for being vertices of the polygon.

Assumption 1 All upper bounds $u_{j}$ for $j \in B$ are equal to one, i.e., we have $u_{j}=1$ for all $j \in B$.

Lemma 11 Let $\sum_{j \in N} \alpha_{j} s_{j} \geq 1$ be facet defining for $\operatorname{conv}\left(P_{I}\right)$ and in standard form. Assume $\alpha_{j}>0$ for all $j \in U$. All vertices of $L_{\alpha}$ are of one of the following two forms

$$
\text { (i) } f+\sum_{j \in I} r^{j}, \quad \text { where } I \subseteq B
$$

(ii) $f+\sum_{j \in I} r^{j}+\frac{1-\sum_{j \in I} \alpha_{j}}{\alpha_{k}} r^{k}, \quad$ where $I \subseteq B$ and $k \in N \backslash I$.

Furthermore, if cone $e_{i \in U} r^{i}=\mathbb{R}^{2}$, then all vertices of $L_{\alpha}$ are of the form (29). 
Proof: By definition $L_{\alpha}$ is the projection of the polyhedron

$$
L_{\alpha}^{s}:=\left\{(x, s) \in P: \sum_{j \in N} \alpha_{j} s_{j} \leq 1\right\}
$$

onto the space of the $x$ variables. From LP theory, every vertex of $L_{\alpha}$ is the projection of a vertex of $L_{\alpha}^{s}$. Let $(\bar{x}, \bar{s})$ be a vertex of $L_{\alpha}^{s}$. We first distinguish two cases based on whether $\sum_{j=1}^{n} \alpha_{j} \bar{s}_{j} \leq 1$ is at equality or not.

Case $1 \sum_{j=1}^{n} \alpha_{j} \bar{s}_{j}<1$.

If there exists $j \in B$ such that $0<\bar{s}_{j}<u_{j}$, then there exists $\epsilon>0$ such that $\left(\bar{x}-\epsilon r^{j}, \bar{s}-\epsilon e_{j}\right),\left(\bar{x}+\epsilon r^{j}, \bar{s}+\epsilon e_{j}\right) \in L_{\alpha}^{s}$, which proves that $(\bar{x}, \bar{s})$ is not a vertex of $L_{\alpha}^{s}$, and therefore $\bar{x}$ is not a vertex of $L_{\alpha}$. Hence it follows that, in this case, every vertex of $L_{\alpha}^{s}$ must be of the form (28).

Case $2 \sum_{j=1}^{n} \alpha_{j} \bar{s}_{j}=1$.

A vertex $v$ of $L_{\alpha}^{s}$ is determined by setting $n$ linearly independent inequalities from the description of $L_{\alpha}^{s}$ to equality. A vertex of $L_{\alpha}^{s}$ is therefore determined by $n-1$ lower and upper bound constraints and the equality $\sum_{j \in N} \alpha_{j} s_{j}=1$.

Finally, if cone $e_{i \in U} r^{i}=\mathbb{R}^{2}$, then there exist $i_{1}, i_{2}, i_{3} \in U$ such that $\sigma_{i_{1}} r^{i_{1}}+$ $\sigma_{i_{2}} r^{i_{2}}+\sigma_{i_{3}} r^{i_{3}}=0$, with $\sigma_{i_{k}} \geq 0$ for $k=1,2,3$. We claim that this implies that Case 1 cannot occur. Indeed, consider $(\bar{x}, \bar{s}) \in L_{\alpha}^{s}$ with $\sum_{j=1}^{n} \alpha_{j} \bar{s}_{j}<1$. Then there exists $\epsilon>0$ such that $\left(\bar{x}+\epsilon \sigma_{i_{k}} r^{i_{k}}, \bar{s}+\epsilon \sigma_{i_{k}} e_{i_{k}}\right) \in L_{\alpha}^{s}$ for $k=1,2,3$, which shows that $(\bar{x}, \bar{s})$ is a convex combination of points in $L_{\alpha}^{s}$.

In the following we assume cone $i \in U r^{i}=\mathbb{R}^{2}$ in order to reduce the number of cases to consider. We therefore only consider vertices of $L_{\alpha}$ of the form (29). We next prove that, for vertices of $L_{\alpha}$ of the form (29) generated from an unbounded ray $r^{k}$ with $k \in U$, we only need to consider $k \in I^{U}(x)$ for some vertex $x$ of $\operatorname{conv}\left(X_{\alpha}\right)$.

Lemma 12 Let $\sum_{j \in N} \alpha_{j} s_{j} \geq 1$ be facet defining for $\operatorname{conv}\left(P_{I}\right)$ and in standard form. Assume $\alpha_{j}>0$ for $j \in U$. Let $I \subseteq B$ satisfy $0 \leq \sum_{i \in I} \alpha_{i}<1$, and let $\{j, k, l\} \subseteq N$ be such that $k, l \in U$ and $r^{j} \in \operatorname{cone}\left\{r^{k}, r^{l}\right\}$. If $\{j, k, l\}$ satisfies the linear dependence property wrt. $\alpha$, then $f+\sum_{i \in I} r^{i}+\frac{1-\sum_{i \in I} \alpha_{i}}{\alpha_{j}} r^{j}$ is not a vertex of $L_{\alpha}$.

Proof: From linear dependence property there exist $\sigma_{k}, \sigma_{l} \geq 0$ such that $r^{j}=$ $\sigma_{k} r^{k}+\sigma_{l} r^{l}$ together with $\alpha_{j}=\sigma_{k} \alpha_{k}+\sigma_{l} \alpha_{l}$. Hence we have

$$
\begin{aligned}
f+\sum_{i \in I} r^{i}+\frac{1-\sum_{i \in I} \alpha_{i}}{\alpha_{j}} r^{j}=f & +\sum_{i \in I} r^{i}+\frac{1-\sum_{i \in I} \alpha_{i}}{\sigma_{k} \alpha_{k}+\sigma_{l} \alpha_{l}}\left(\sigma_{k} r^{k}+\sigma_{l} r^{l}\right) \\
=\frac{\sigma_{k} \alpha_{k}}{\sigma_{k} \alpha_{k}+\sigma_{l} \alpha_{l}}\left(f+\sum_{i \in I} r^{i}\right. & \left.+\frac{1-\sum_{i \in I} \alpha_{i}}{\alpha_{k}} r^{k}\right) \\
& +\frac{\sigma_{l} \alpha_{l}}{\sigma_{k} \alpha_{k}+\sigma_{l} \alpha_{l}}\left(f+\sum_{i \in I} r^{i}+\frac{1-\sum_{i \in I} \alpha_{i}}{\alpha_{l}} r^{l}\right)
\end{aligned}
$$

which proves that it is the convex combination of two points of $L_{\alpha}$. 
Lemma 12 shows that rays $r^{k}$ with $k \in U$ that are in $\operatorname{cone}\left\{r^{i}, r^{j}\right\}$ where $i, j \in U$, and with $\{i, j, k\}$ satisfying the linear dependence property wrt. $\alpha$, do not generate vertices of $L_{\alpha}$ of the form (29). From this lemma we conclude that it is enough to consider unbounded rays involved in maximal representations (rays in some $I^{U}(x)$ ) and the bounded rays to construct the vertices of $L_{\alpha}$. Note that this corresponds to the indices appearing in the simplified polar (25)(26) in Theorem 1.

\subsection{The unbounded case}

In the unbounded case, there is no linear dependence property (26) involving a bounded ray, and therefore the simplified polar only consists of (25), and this gives the same number of equations as the number of integer points with at least one tight representation. We conclude that the simplified polar, which is uniquely solvable, contains either three rows and three rays or four rows and four rays. The vertices of $L_{\alpha}$ are given by $f+\frac{1}{\alpha_{i}} r^{i}$ as stated by Lemma 11 . This leads to either a triangle or a quadrilateral. We can therefore obtain the coefficients of a facet from the polygon $L_{\alpha}$. In the unbounded case, it is explicitly given by the ratio of the norm of a ray $i$ divided by the distance to which the ray $i$ intersects $L_{\alpha}$. See [1] for more details on the geometry of the unbounded case.

\subsection{The one edge case}

In the remainder of this section, we consider the case when $B=\{e\}$, and we call the only bounded ray $r^{e}$ for the edge. The situation is slightly different in this case. There is still one maximal tight representation (25) for each integer point, and some linear dependence equations (27) (that we discard as in Theorem 1) and (26) (that we keep).

The difference comes from the fact that the edge may occur in several distinct linear dependencies (26). This number is however limited by two as we will prove in the following lemmas.

Lemma 13 Let $\sum_{i \in N} \alpha_{i} s_{i} \geq 1$ be facet defining for $\operatorname{conv}\left(P_{I}\right)$ and in standard form. Consider two points $x, y \in \mathbb{Z}^{2}$ such that $\mathcal{S}_{\text {strict }}^{\alpha}(x) \cap \mathcal{S}_{\text {strict }}^{\alpha}(y) \supseteq\{i, e\}$ with $i \in U$. Then $\mathcal{S}_{\text {strict }}^{\alpha}(x)=\mathcal{S}_{\text {strict }}^{\alpha}(y)$.

Proof: We only prove $\mathcal{S}_{\text {strict }}^{\alpha}(x) \subseteq \mathcal{S}_{\text {strict }}^{\alpha}(y)$. The proof of the other inclusion is symmetric. Let $j \in \mathcal{S}_{\text {strict }}^{\alpha}(x)$. Hence $\{i, j, e\}$ satisfies the linear dependence property wrt. $\alpha$, and therefore there exist $\sigma_{i}, \sigma_{j}, \sigma_{e} \in \mathbb{R}$ with $\sigma_{j}>0$ such that $\sigma_{i} r^{i}+\sigma_{j} r^{j}+\sigma_{e} r^{e}=0$ and $\sigma_{i} \alpha_{i}+\sigma_{j} \alpha_{j}+\sigma_{e} \alpha_{e}=0$. Let $t^{*} \in T_{\alpha}(y)$ denote a representation of $y$ that satisfies (11). We have $\{i, e\} \subseteq T_{\text {strict }}^{*}$. We therefore have $t^{*}+\epsilon\left(\sigma_{i} \mathbf{e}_{i}+\sigma_{j} \mathbf{e}_{j}+\sigma_{e} \mathbf{e}_{e}\right) \in T_{\alpha}(y)$ for $\epsilon>0$ small enough, where $\mathbf{e}_{i}, \mathbf{e}_{j}$ and $\mathbf{e}_{e}$ are unit vectors. This implies $j \in \mathcal{S}_{\text {strict }}^{\alpha}(y)$.

The previous lemma implies that if $e$ is involved in two linear dependence properties, then the vectors must be different. Furthermore, if $e$ is involved in 
two linear dependence properties, then it cannot belong to the corresponding cones as the next lemma shows.

Lemma 14 Let $\sum_{i \in N} \alpha_{i} s_{i} \geq 1$ be facet defining for $\operatorname{conv}\left(P_{I}\right)$ and in standard form. Consider $x, y \in \mathbb{Z}^{2}$ such that $\mathcal{S}_{\text {strict }}^{\alpha}(x) \cap \mathcal{S}_{\text {strict }}^{\alpha}(y)=\{e\}$. Then $r^{e} \notin$ $\operatorname{cone}\left\{r^{j}: j \in \mathcal{S}_{\text {strict }}^{\alpha}(x) \backslash\{e\}\right\}$ and $r^{e} \notin \operatorname{cone}\left\{r^{j}: j \in \mathcal{S}_{\text {strict }}^{\alpha}(y) \backslash\{e\}\right\}$.

Proof: Assume for a contradiction that $r^{e} \in \operatorname{cone}\left\{r^{j}: j \in \mathcal{S}_{\text {strict }}^{\alpha}(x) \backslash\{e\}\right\}$. Then there exists $k, l \in \mathcal{S}_{\text {strict }}^{\alpha}(x)$ and $\sigma_{k}, \sigma_{l} \geq 0$ such that $r^{e}=\sigma_{k} r^{k}+\sigma_{l} r^{l}$ and $\alpha_{e}=\sigma_{k} \alpha_{k}+\sigma_{l} \alpha_{l}$. Let $t^{*} \in T_{\alpha}(y)$ satisfy $0<t_{e}^{*}<1$. We have $t^{*}+\epsilon\left(-\mathbf{e}_{e}+\right.$ $\left.\sigma_{k} \mathbf{e}_{k}+\sigma_{l} \mathbf{e}_{l}\right) \in T_{\alpha}(y)$ for $\epsilon>0$ small enough, where $\mathbf{e}_{e}, \mathbf{e}_{k}$ and $\mathbf{e}_{l}$ are unit vectors. We conclude that $k, l \in \mathcal{S}_{\text {strict }}^{\alpha}(y)$ which is a contradiction with the hypothesis that $\mathcal{S}_{\text {strict }}^{\alpha}(x) \cap \mathcal{S}_{\text {strict }}^{\alpha}(y)=\{e\}$.

The next lemma shows that the edge cannot be involved in three linear dependence properties.

Lemma 15 Let $\sum_{i \in N} \alpha_{i} s_{i} \geq 1$ be facet defining for $\operatorname{conv}\left(P_{I}\right)$ and in standard form. Consider $x^{1}, x^{2}, x^{3} \in \mathbb{Z}^{2}$ such that $\mathcal{S}_{\text {strict }}^{*}\left(x^{i}, \alpha\right) \cap \mathcal{S}_{\text {strict }}^{*}\left(x^{j}, \alpha\right) \supseteq\{e\}$ for all pairs $\{i, j\} \subset\{1,2,3\}$. Then there exists at least one pair $\{i, j\} \subset\{1,2,3\}$ with $i \neq j$ such that $\mathcal{S}_{\text {strict }}^{\alpha}\left(x^{i}\right)=\mathcal{S}_{\text {strict }}^{\alpha}\left(x^{j}\right)$.

Proof: From Lemma 13, we know that if $\mathcal{S}_{\text {strict }}^{\alpha}\left(x^{i}\right) \cap \mathcal{S}_{\text {strict }}^{\alpha}\left(x^{j}\right) \supsetneq\{e\}$ for some $i \neq j$, then $\mathcal{S}_{\text {strict }}^{\alpha}\left(x^{i}\right)=\mathcal{S}_{\text {strict }}^{\alpha}\left(x^{j}\right)$. In this case the result therefore follows.

We may therefore assume $\mathcal{S}_{\text {strict }}^{\alpha}\left(x^{i}\right) \cap \mathcal{S}_{\text {strict }}^{\alpha}\left(x^{j}\right)=\{e\}$ for all $\{i, j\} \subset$ $\{1,2,3\}$. Lemma 7 and Lemma 14 imply $-r^{e}, r^{e} \notin \operatorname{cone}\left\{r^{k}: k \in \mathcal{S}_{\text {strict }}^{\alpha}\left(x^{i}\right)\right\}$ for $i=1,2,3$. Therefore the line $\operatorname{span}\{e\}$ separates $\mathbb{R}^{2}$ in two halfspaces such that there exists two indices $\{p, q\} \subset\{1,2,3\}$ with cone $\left\{r^{j}: j \in \mathcal{S}_{\text {strict }}^{\alpha}\left(x^{p}\right)\right\}$ and cone $\left\{r^{j}: j \in \mathcal{S}_{\text {strict }}^{\alpha}\left(x^{q}\right)\right\}$ belonging to the same halfspace. We claim $\mathcal{S}_{\text {strict }}^{\alpha}\left(x^{p}\right)=\mathcal{S}_{\text {strict }}^{\alpha}\left(x^{q}\right)$. Since they belong to the same halfspace, we can write $\operatorname{cone}\left\{r^{j}: j \in \mathcal{S}_{\text {strict }}^{\alpha}\left(x^{p}\right) \cup \mathcal{S}_{\text {strict }}^{\alpha}\left(x^{q}\right) \cup\{e\}\right\}=\operatorname{cone}\left\{r^{e}, r^{i}\right\}$ with wlog $i \in \mathcal{S}_{\text {strict }}^{\alpha}\left(x^{p}\right)$. We claim that also $i \in \mathcal{S}_{\text {strict }}^{\alpha}\left(x^{q}\right)$, which implies $\{i, e\} \subseteq$ $\mathcal{S}_{\text {strict }}^{\alpha}\left(x^{p}\right) \cap \mathcal{S}_{\text {strict }}^{\alpha}\left(x^{q}\right)$, and therefore Lemma 13 shows $\mathcal{S}_{\text {strict }}^{\alpha}\left(x^{p}\right)=\mathcal{S}_{\text {strict }}^{\alpha}\left(x^{q}\right)$.

Let $t^{*} \in T_{\alpha}\left(x^{q}\right)$ and $v^{*} \in T_{\alpha}\left(x^{p}\right)$ be tight representations of $x^{q}$ and $x^{p}$ respectively that satisfy (11). For any $j \in \mathcal{S}_{\text {strict }}^{\alpha}\left(x^{p}\right) \cup \mathcal{S}_{\text {strict }}^{\alpha}\left(x^{q}\right)$, there exists $\sigma_{i}, \sigma_{e} \geq 0$ such that $r^{j}=\sigma_{i} r^{i}+\sigma_{e} r^{e}$. Now, there exist $\delta, \epsilon>0$ such that $t^{*}+$ $\epsilon\left(-\mathbf{e}_{j}+\sigma_{i} \mathbf{e}_{i}+\sigma_{e} \mathbf{e}_{e}\right)$ is a valid representation of $x^{q}$ and $v^{*}+\delta\left(\mathbf{e}_{j}-\sigma_{i} \mathbf{e}_{i}-\sigma_{e} \mathbf{e}_{e}\right)$ is a valid representation of $x^{p}$. Therefore we have $\sum_{\bar{i} \in N} \alpha_{\bar{i}} t_{\bar{i}}^{*}=1$ and $\sum_{\bar{i} \in N} \alpha_{\bar{i}} t_{\bar{i}}^{*}+$ $\epsilon\left(-\alpha_{j}+\sigma_{i} \alpha_{i}+\sigma_{e} \alpha_{e}\right) \geq 1$ from which we conclude $\alpha_{j} \leq \sigma_{i} \alpha_{i}+\sigma_{e} \alpha_{e}$. Similarly we have $\sum_{\bar{i} \in N} \alpha_{\bar{i}} v_{\bar{i}}^{*}=1$ and $\sum_{\bar{i} \in N} \alpha_{\bar{i}} v_{\bar{i}}^{*}+\delta\left(\alpha_{j}-\sigma_{i} \alpha_{i}-\sigma_{e} \alpha_{e}\right) \geq 1$ from which we conclude $\alpha_{j} \geq \sigma_{i} \alpha_{i}+\sigma_{e} \alpha_{e}$. Therefore $\alpha_{j}=\sigma_{i} \alpha_{i}+\sigma_{e} \alpha_{e}$ from which we conclude that $t^{*}+\epsilon\left(-\mathbf{e}_{j}+\sigma_{i} \mathbf{e}_{i}+\sigma_{e} \mathbf{e}_{e}\right) \in T_{\alpha}\left(x^{q}\right)$ which proves that $i \in \mathcal{S}_{\text {strict }}^{\alpha}\left(x^{q}\right)$.

Corollary 4 Let $\sum_{i \in N} \alpha_{i} s_{i} \geq 1$ be facet defining for $\operatorname{conv}\left(P_{I}\right)$ and in standard form. If $\{e, 1,2\}$ and $\{e, 3,4\}$ satsify the linear dependence property wrt. $\alpha$ and cone $\left\{r^{e}, r^{1}, r^{2}, r^{3}, r^{4}\right\} \neq \mathbb{R}^{2}$, then $\{e, 1,2,3,4\}$ satisifes the linear dependence property wrt. $\alpha$. 
Proof: The proof is identical to the second part of the proof of Lemma 15.

In the unbounded case, the vertices of $L_{\alpha}$ are given by $f+\frac{1}{\alpha_{i}}$. When an edge is present, each ray $r^{i}$ involved in maximal representations gives rise to two potential vertices $f+\frac{1}{\alpha_{i}} r^{i}$ and $f+r^{e}+\frac{1-\alpha_{e}}{\alpha_{i}} r^{i}$. Observe that if $\alpha_{e}>1$, each ray gives rise to one potential vertex. This is therefore essentially the same situation as in the unbounded case. Therefore, in this section, we only consider facet-defining inequalities in standard form with $\alpha_{i}>0$ for all $i \in U$ and $\alpha_{e} \leq 1$. We next present results that enable us to rule out some potential vertices of $L_{\alpha}$.

Lemma 16 Let $\sum_{i \in N} \alpha_{i} s_{i} \geq 1$ be facet defining for $\operatorname{conv}\left(P_{I}\right)$ and in standard from. Suppose $\alpha_{i}>0$ for all $i \in U$ and $\alpha_{e}<1$. If $r^{e} \in \operatorname{cone}\left\{r^{j}, r^{k}\right\}$ and $\{e, j, k\}$ satisfies the linear dependence property wrt. $\alpha$, then $f+r^{e}+\frac{1-\alpha_{e}}{\alpha_{i}} r^{i}$ is not a vertex of $L_{\alpha}$ for any $i \in U$.

Proof: There are two cases to analyze. We first prove the lemma for $i=j$ (the case $i=k$ is similar), and then for $i \neq j, k$.

(i) We first prove $f+r^{e}+\frac{1-\alpha_{e}}{\alpha_{j}} r^{j}$ is not a vertex when $i=j$.

We have $r^{e}=\sigma_{j} r^{j}+\sigma_{k} r^{k}$ with $\sigma_{j}, \sigma_{k} \geq 0$ and $\alpha_{e}=\sigma_{j} \alpha_{j}+\sigma_{k} \alpha_{k}$ by linear dependence property. Therefore

$$
\begin{aligned}
f+r^{e}+\frac{1-\alpha_{e}}{\alpha_{j}} r^{j} & =f+\sigma_{j} r^{j}+\sigma_{k} r^{k}+\frac{1-\sigma_{j} \alpha_{j}-\sigma_{k} \alpha_{k}}{\alpha_{j}} r^{j} \\
& =f+\left(1-\sigma_{k} \alpha_{k}\right) \frac{r^{j}}{\alpha_{j}}+\left(\sigma_{k} \alpha_{k}\right) \frac{r^{k}}{\alpha_{k}} \\
& =\left(1-\sigma_{k} \alpha_{k}\right)\left(f+\frac{r^{j}}{\alpha_{j}}\right)+\left(\sigma_{k} \alpha_{k}\right)\left(f+\frac{r^{k}}{\alpha_{k}}\right)
\end{aligned}
$$

which proves that $f+r^{e}+\frac{1-\alpha_{e}}{\alpha_{j}} r^{j}$ is not a vertex of $L_{\alpha}$ (observe that $0 \leq \sigma_{k} \alpha_{k} \leq$ 1 since we assumed $\left.\alpha_{e} \leq 1\right)$.

(ii) We next prove $f+r^{e}+\frac{1-\alpha_{e}}{\alpha_{i}} r^{i}$ is not a vertex when $i \neq j, k$.

We have

$$
\begin{aligned}
f+r^{e}+ & \frac{1-\alpha_{e}}{\alpha_{i}} r^{i}=f+\sigma_{j} r^{j}+\sigma_{k} r^{k}+\frac{1-\sigma_{j} \alpha_{j}-\sigma_{k} \alpha_{k}}{\alpha_{i}} r^{i} \\
& =\left(\sigma_{j} \alpha_{j}\right)\left(f+\frac{r^{j}}{\alpha_{j}}\right)+\left(\sigma_{k} \alpha_{k}\right)\left(f+\frac{r^{k}}{\alpha_{k}}\right)+\left(1-\sigma_{j} \alpha_{j}-\sigma_{k} \alpha_{k}\right)\left(f+\frac{r^{i}}{\alpha_{i}}\right)
\end{aligned}
$$

which shows $f+r^{e}+\frac{1-\alpha_{e}}{\alpha_{i}} r^{i}$ is a convex combination of three points of $L_{\alpha}$.

Lemma 17 Let $\sum_{i \in N} \alpha_{i} s_{i} \geq 1$ be facet defining for $\operatorname{conv}\left(P_{I}\right)$ and in standard form. Suppose $\alpha_{i}>0$ for all $i \in U$ and $\alpha_{e}<1$, and let $j, k$ be such that $r^{k} \in \operatorname{cone}\left\{r^{j}, r^{e}\right\}$. Then 
(i) If $\{e, j, k\}$ satisfies the linear dependence property wrt. $\alpha$, then $f+r^{e}+$ $\frac{1-\alpha_{e}}{\alpha_{j}} r^{j}$ and $f+\frac{1}{\alpha_{k}} r^{k}$ are convex combinations of $f+r^{e}+\frac{1-\alpha_{e}}{\alpha_{k}} r^{k}$ and $f+\frac{1}{\alpha_{j}} r^{j}$.

(ii) If $\{e, j, k\}$ does not satisfy the linear dependence property wrt. $\alpha$, then either $f+r^{e}+\frac{1-\alpha_{e}}{\alpha_{j}} r^{j}$ or $f+\frac{1}{\alpha_{k}} r^{k}$ is not a vertex of $L_{\alpha}$.

Proof: (i) We have $r^{k}=\sigma_{j} r^{j}+\sigma_{e} r^{e}$ with $\sigma_{j}, \sigma_{e} \geq 0$ and also $\alpha_{k}=\sigma_{j} \alpha_{j}+\sigma_{e} \alpha_{e}$. It can be verified that

$$
f+\frac{r^{k}}{\alpha_{k}}=\left(1-\frac{\sigma_{e}}{\sigma_{e}+\sigma_{j} \alpha_{j}}\right)\left(f+\frac{r^{j}}{\alpha_{j}}\right)+\left(\frac{\sigma_{e}}{\sigma_{e}+\sigma_{j} \alpha_{j}}\right)\left(f+r^{e}+\frac{1-\alpha_{e}}{\alpha_{k}} r^{k}\right) .
$$

Observe that the coefficients in the above combination are in the interval $[0,1]$. It can also be verified that

$$
\begin{aligned}
f+r^{e}+\frac{1-\alpha_{e}}{\alpha_{j}} r^{j}=\left(1-\frac{\sigma_{j} \alpha_{j}+\sigma_{e} \alpha_{e}}{\sigma_{j} \alpha_{j}+\sigma_{e}}\right) & \left(f+\frac{r^{j}}{\alpha_{j}}\right) \\
& +\left(\frac{\sigma_{j} \alpha_{j}+\sigma_{e} \alpha_{e}}{\sigma_{j} \alpha_{j}+\sigma_{e}}\right)\left(f+r^{e}+\frac{1-\alpha_{e}}{\alpha_{k}} r^{k}\right) .
\end{aligned}
$$

The coefficients in the above combination belong to $[0,1]$ since $\alpha_{e} \leq 1$.

(ii) Observe that $r^{k}=\sigma_{j} r^{j}+\sigma_{e} r^{e}$ and either

$$
\begin{aligned}
& \alpha_{k}>\sigma_{j} \alpha_{j}+\sigma_{e} \alpha_{e} \quad \text { or } \\
& \alpha_{k}<\sigma_{j} \alpha_{j}+\sigma_{e} \alpha_{e}
\end{aligned}
$$

In the case of (30), $f+\frac{1}{\alpha_{k}} r^{k}$ is not a vertex of $L_{\alpha}$. Indeed there exists $\epsilon>0$ such that $\left(\frac{1}{\alpha_{k}}-\epsilon\right)>0$ and

$$
f+\frac{1}{\alpha_{k}} r^{k}=f+\left(\frac{1}{\alpha_{k}}-\epsilon\right) r^{k}+\epsilon \sigma_{j} r^{j}+\epsilon \sigma_{e} r^{e}
$$

with

$$
\alpha_{k}\left(\frac{1}{\alpha_{k}}-\epsilon\right)+\alpha_{j} \epsilon \sigma_{j}+\alpha_{e} \epsilon \sigma_{e}=1+\epsilon\left(\sigma_{j} \alpha_{j}+\sigma_{e} \alpha_{e}-\alpha_{k}\right)<1 .
$$

Therefore $f+\frac{1}{\alpha_{k}} r^{k}$ is not a vertex of $L_{\alpha}$, since a point $f+\sum_{j \in N} s_{j} r^{j}$ satisfying $\sum_{j \in N} \alpha_{j} s_{j}<1$ cannot be a vertex of $L_{\alpha}$.

The same kind of argument works for $f+r^{e}+\frac{1-\alpha_{e}}{\alpha_{j}} r^{j}$ in the case of (31)

We are now able to classify the geometry of all facets occurring in a problem with one edge. To this end, we distinguish whether or not three or four integer points have tight representations. We then consider three subcases depending on the number of occurences of the edge in a linear dependence property.

Three integer points 
(i) The edge is involved in no linear dependence property.

This case is impossible, because then there are three equations (involving $\left.\alpha_{e}\right)$ that give a uniquely solvable system. Hence there are at most two rays remaining. This is a contradiction since they do not span $\mathbb{R}^{2}$.

(ii) The edge is involved in one linear dependence property, e.g. $\{e, j, k\}$ satisfies the linear dependence property.

Two main cases occur. If $r^{e} \in \operatorname{cone}\left\{r^{j}, r^{k}\right\}$, then by Lemma 16, all vertices are of the form $f+\frac{1}{\alpha_{i}} r^{i}$ which makes three vertices (see Fig. 3(a) for an example). If $r^{e} \notin \operatorname{cone}\left\{r^{j}, r^{k}\right\}$, then $f+\frac{1}{\alpha_{j}} r^{j}$ and $f+r^{e}+\frac{1-\alpha_{e}}{\alpha_{k}} r^{k}$ are vertices of $L_{\alpha}$ by Lemma 17(i). The third ray $r^{i}$ involved in the reduced polar induces a maximum of two vertices $f+\frac{1}{\alpha_{i}} r^{i}$ and $f+r^{e}+\frac{1-\alpha_{e}}{\alpha_{i}} r^{i}$. We therefore have at most four vertices in total (see Fig. 3(b) for an example).

(iii) The edge is involved in two linear dependencies.

From Lemma 13, we know that this implies that four rays are involved in the maximal tight representations. In total, we therefore have 5 equations and 4 rays and one edge. Let $r^{i}, r^{j}, r^{k}, r^{l}$ be the four rays involved in the linear dependence properties. We must have, from Lemma 14, wlog $r^{j} \in \operatorname{cone}\left\{r^{i}, r^{e}\right\}$ and $r^{l} \in \operatorname{cone}\left\{r^{k}, r^{e}\right\}$. From Lemma 17(i), there are four vertices $f+\frac{1}{\alpha_{i}} r^{i}, f+r^{e}+\frac{1-\alpha_{e}}{\alpha_{j}} r^{j}, f+\frac{1}{\alpha_{i}} r^{k}$, and $f+r^{e}+\frac{1-\alpha_{e}}{\alpha_{l}} r^{l}$ (see Fig. $3(\mathrm{c}))$.

\section{Four integer points}

(i) The edge is involved in no linear dependence property

We therefore have four equations, one edge and three rays, $r^{i}, r^{j}, r^{k}$. There must exist a pair, say $i, j$, such that $r^{j} \in \operatorname{cone}\left\{r^{i}, r^{e}\right\}$. By Lemma 17(ii), we have that either $f+r^{e}+\frac{1-\alpha_{e}}{\alpha_{i}} r^{i}$ or $f+\frac{1}{\alpha_{j}} r^{j}$ is not a vertex. We therefore have a maximum of five vertices (see Fig. 4(a)).

(ii) The edge is involved in one linear dependence property.

We therefore have five equations, one edge and four rays. Let $\{i, j, e\}$ satisfy the linear dependence property, and $r^{k}, r^{l}$ be the two remaining rays. If $r^{e} \in \operatorname{cone}\left\{r^{i}, r^{j}\right\}$, by Lemma 16, all vertices are of the form $f+\frac{1}{\alpha_{t}} r^{t}$.

Let us assume $r^{j} \in \operatorname{cone}\left\{r^{i}, r^{e}\right\}$. Then $f+\frac{1-\alpha_{e}}{\alpha_{i}} r^{i}$ and $f+\frac{1}{\alpha_{j}} r^{j}$ are not vertices of $L_{\alpha}$. If we consider the line $\lambda r^{e}, \lambda \in \mathbb{R}$, it separates the plane into two half-planes $H_{1}, H_{2}$. Let us assume that $r^{i}, r^{j} \in H_{1}$. At least one among $r^{k}, r^{l}$ belongs to $H_{2}$. The other ray, say $r^{l}$, is either in $H_{1}$ or in $H_{2}$. If it is in $H_{2}$, then $r^{k} \in \operatorname{cone}\left\{r^{l}, r^{e}\right\}$ or $r^{l} \in \operatorname{cone}\left\{r^{k}, r^{e}\right\}$. In both cases, it creates at most three more vertices (by Lemma 17(ii)) and yields five vertices in total (see Fig. 4(b)). If $r^{l} \in H_{1}$, then we have in one case $r^{i}, r^{j} \in$ cone $\left\{r^{l}, r^{e}\right\}$ which implies that either $f+r^{e}+\frac{1-\alpha_{e}}{\alpha_{l}} r^{l}$ is not a vertex (but makes 5 vertices in total) or $f+\frac{r^{i}}{\alpha_{i}}$ is not a vertex ( 5 vertices in total). We obtain similarly 5 vertices in the other case if $r^{l} \in \operatorname{cone}\left\{r^{j}, r^{e}\right\}$. 


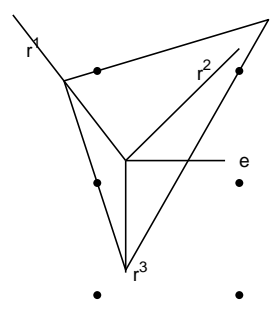

(a) 3 points, one lin. dep.

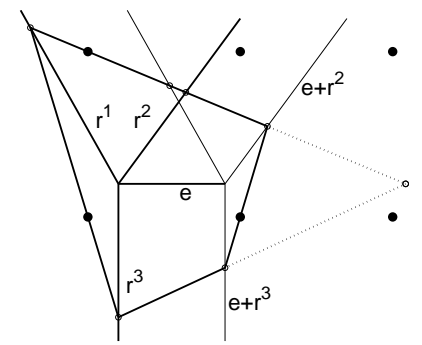

(b) 3 points, one lin. dep.

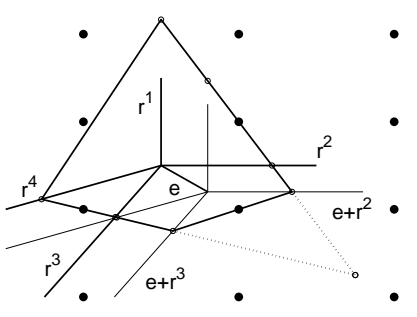

(c) 3 points, two lin. dep.

Figure 3: The possible cases with three integer points

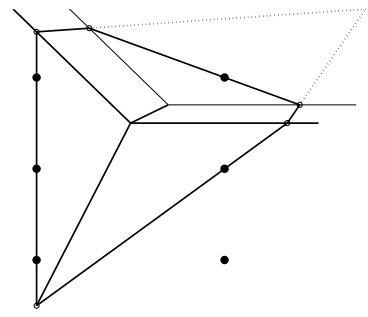

(a) 4 points, no lin. dep.

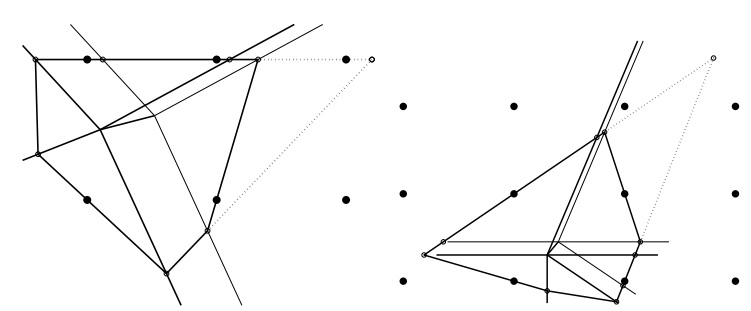

(b) 4 points, one lin. dep.

(c) 4 points, two lin. dep.

Figure 4: The possible cases with four integer points

(iii) The edge is involved in two linear dependence properties.

We then have six equations and five rays $\left\{r^{i}, r^{j}, r^{k}, r^{l}, r^{m}\right\}$ where $\{i, j, e\}$ and $\{k, l, e\}$ satisfy the linear dependence property. From Lemma 14 and Corollary 4, we have wlog $r^{j} \in \operatorname{cone}\left\{r^{i}, r^{e}\right\}$ and $r^{l} \in \operatorname{cone}\left\{r^{k}, r^{e}\right\}$. This implies four vertices involving $i, j, k, l$ from Lemma 17(i). Now $r^{m}$ is either on the same side as cone $\left\{r^{i}, r^{j}\right\}$ with respect to $r^{e}$ or as cone $\left\{r^{k}, r^{l}\right\}$. Following the same reasoning as in the previous case, we also conclude to a maximum of five vertices (see Fig. 4(c)).

When an edge is present, the vertices of $L_{\alpha}$ are not necessarily located on $f+\lambda r^{i}$. They may also be located on $f+u_{e} r^{e}+\lambda r^{i}$. This observation allows us to determine the coefficients of an inequality from a polygon $L_{\alpha}$. For every ray $i$ such that a vertex of $L_{\alpha}$ lies on $f+\lambda r^{i}, \lambda \geq 0$, the coefficient $\alpha_{i}$ can be obtained as before.

The coefficient $\alpha_{e}$ can be obtained as usual if the edge intersects the polygon $L_{\alpha}$ (in this case the bound is irrelevant). If the edge does not intersect $L_{\alpha}$, there exist two sides of the polygon that meet on the line $f+\lambda r^{e}, \lambda \geq 0$. This "hidden vertex" of $L_{\alpha}$ determines the coefficient $\alpha_{e}$. It is shown by the intersection of two dotted lines on Fig. 3 and 4.

Finally, for every ray $i$ such that a vertex of $L_{\alpha}$ is on $f+u_{e} r^{e}+\lambda r^{i}, \lambda \geq 0$, the coefficient $\alpha_{i}$ is given by $\left(1-u_{e} \alpha_{e}\right)$ multiplied by the ratio of the norm of 
the ray $r^{i}$ divided by $\lambda$ where $f+u_{e} r^{e}+\lambda r^{i}$ is a vertex of $L_{\alpha}$.

Some rays do not determine a vertex of $L_{\alpha}$. The coefficient of such a ray $i$ can be obtained by one of the two previous ways. It is essentially obtained through the maximum of the two previous methods.

\subsection{Conclusion}

In this paper, we have shown that the presence of finite upper bounds complicates the geometric description of the facet structure of $\operatorname{conv}\left(P_{I}\right)$. For the case of one bounded variable, we managed to give a complete description of the mixed integer hull. We found that for most inequalities that have $\alpha_{e}<1$, stronger inequalities can be obtained than in the unbounded case. Consider, for example, the inequality shown in Fig. 3 (b). Let us forget for a while that the variable $s_{e}$ is bounded, but suppose that we can still obtain the coefficients from the geometry of the bounded problem. In that case, the natural polygon obtained is the large triangle supported by the solid and dotted lines. We see that this polygon includes the integer point $(1,0)$ in its interior. This proves that the inequality could not be obtained from the unbounded relaxation. The fact that we consider the bound explicitly allows us to strengthen the coefficient $\alpha_{e}$.

\section{Acknowledgements}

We would like to thank two anonymous referees for their helpful comments.

This text presents research results of the Belgian Program on Interuniversity Poles of Attraction initiated by the Belgian State, Prime Minister's Office, Science Policy Programming. The scientific responsibility is assumed by the authors.

\section{References}

[1] Andersen, K., Louveaux, Q., Weismantel, R., Wolsey, L.A.: Inequalities from two rows of a simplex tableau. In: M. Fischetti, D.P. Williamson (eds.) IPCO, Lecture Notes in Computer Science, vol. 4513, pp. 1-15. Springer (2007)

[2] Arkinstall, J.R.: Minimal requirements for minkowskis theorem in the plane. I. Bull. Austral. Math. Soc (22) (1980)

[3] Balas, E., Ceria, S., Cornuéjols, G.: A lift-and-project cutting plane algorithm for mixed 0/1 programs. Mathematical Programming 58, 295-324 (1993)

[4] Cook, W., Kannan, R., Schrijver, A.: Chvátal closures for mixed integer programming problems. Mathematical Programming 47, 155-174 (1990) 
[5] Cornuéjols, G., Margot, F.: On the facets of mixed integer programs with two integer variables and two constraints. Math. Program. to appear (2008)

[6] Gomory, R.: An algorithm for the mixed integer problem. Technical Report RM-2597, The Rand Corporation (1960a)

[7] Gomory, R.E.: Some polyhedra related to combinatorial problems. Linear Algebra and Its Applications 2, 451-558 (1969)

[8] Gomory, R.E., Johnson, E.: Some continuous functions related to corner polyhedra I. Mathematical Programming 3, 23-85 (1972)

[9] Lovász, L.: Geometry of numbers and integer programming. Mathematical Programming, Recent Developments and Applications pp. 177-201 (1989)

[10] Nemhauser, G., Wolsey, L.: Integer and combinatorial optimization. Wiley (1988) 